\title{
Doxorubicin-Bound Hydroxyethyl Starch Conjugate Nanoparticles with pH/Redox Responsive Linkage for Enhancing Antitumor Therapy
}

\author{
Ronghua Tan (i) \\ Danlei Tian \\ Jiaoyan Liu \\ Congcong Wang \\ Ying Wan
}

National Engineering Research Center for Nanomedicine, College of Life Science and Technology, Huazhong University of Science and Technology, Wuhan, 430074, People's Republic of China
Correspondence: Ying Wan

Tel +86 27-87792 I 47

Fax +86 27-87792234

Email ying_wan@hust.edu.cn
Background: Chemotherapeutic drugs used for tumor treatments often show limited efficiency due to their short lifetime, nonspecific delivery, and slow or insufficient intracellular drug release, and also, they can cause severe system or organ toxicity. The development of chemotherapeutic nanomedicines with high efficacy and satisfactory safety still remains a challenge for current tumor chemotherapy.

Methods: A novel type of conjugate was synthesized using hydroxyethyl starch (HES) as a carrier while binding doxorubicin (DOX) onto HES backbone through a $\mathrm{pH} / \mathrm{redox}$ responsive linker containing both disulfide and hydrazone bonds in series. The built conjugates were self-assembled into nanoparticles (NPs) (HES-SS-hyd-DOX NPs) for achieving enhanced antitumor therapy and adequate safety.

Results: HES-SS-hyd-DOX NPs had a certain ability for the tumor-orientated drug accumulation and were capable of releasing DOX itself rather than DOX derivatives. It was found that the $\mathrm{pH} /$ redox responsive linkage enabled the NPs to achieve fast and sufficient intracellular drug release. Based on the tumor-bearing mouse model, antitumor results demonstrated that these NPs were able to inhibit the growth of the advanced tumors with significantly enhanced efficacy when compared to free DOX, and to those conjugate NPs containing only a single responsive or unresponsive bond. Besides, HES-SS-hyd-DOX NPs also showed adequate safety to the normal organs of the treated mice.

Conclusion: The $\mathrm{pH} /$ redox responsive linkage in HES-SS-hyd-DOX was found to play a critical role in mediating the drug accumulation and the fast and sufficient intracellular drug release. The HES-exposed surface of HES-SS-hyd-DOX NPs endowed the NPs with long circulation capability and remarkably reduced the DOX-induced side effects.

Keywords: hydroxyethyl starch, doxorubicin, conjugate nanoparticles, $\mathrm{pH} /$ redox responsive linkage, antitumor therapy

\section{Introduction}

Cancer is one of the most destructive diseases with high incidences and death rates. Despite the varied types of therapeutic modalities available to date, chemotherapy based on anticancer small molecule cytotoxic drugs is the most common approach. ${ }^{1}$ Nevertheless, the efficacy of chemotherapeutic drugs is usually low when they are administered in a free formulation because of their several drawbacks such as the short lifetime in the bloodstream, nonspecific delivery, low bioavailability, and severe system or organ toxicity. ${ }^{1,2}$ Many nano-drug vehicles have thus developed for delivering anti-cancer drugs to prolong their in vivo circulation, enhance their 


\section{Graphical Abstract}

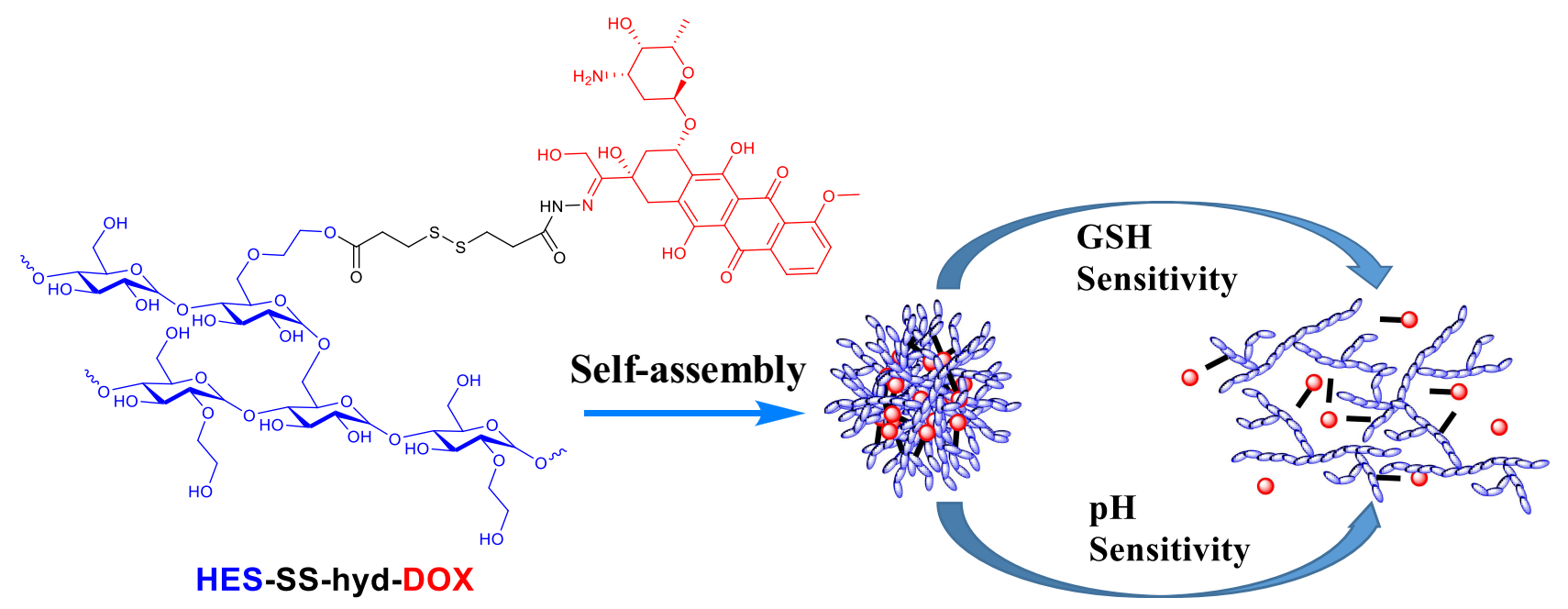

$\mathrm{pH} /$ redox responsive doxorubicin-bound hydroxyethyl starch conjugate nanoparticles.

tumor-orientated accumulation, increase their cancer cellspecific uptake, and alleviate their side effects. ${ }^{3-11}$

Polymeric conjugates used for intravenous drug administration were usually constructed by linking small molecule drugs onto a biodegradable watersoluble polymer through cleavable linkers. It has been reported that around $60 \%$ of drugs in development or clinical applications have low aqueous solubility. ${ }^{4}$ Given that these hydrophobic drugs are delivered as conjugates, their efficiency could be potentially enhanced as conjugates can remarkably improve the aqueous solubility of the hydrophobic drugs and protect them from rapid exclusion from the circulation system. ${ }^{4,6,10}$ By tailoring the backbone of polymer conjugates, the release rate and duration of the bound drug can also be mediated, which offers the opportunity to control the drug release in custom-designed ways. ${ }^{4,10,12}$ In general, the employed polymers for the conjugate backbone have their molecular weight much higher than the renal clearance threshold, and the corresponding conjugates accordingly tend to accumulate in solid tumors via the enhanced permeability and retention (EPR) effect. ${ }^{10,13}$ Besides, many types of conjugates can self-assemble into nanoparticles (NPs) due to the bound hydrophobic drugs, making them safer for in vivo use than the corresponding free drugs as the bound drugs were usually encapsulated inside the NPs. ${ }^{10,12,13}$

Among available biocompatible polymers, hydroxyethyl starch (HES), a biodegradable polysaccharide derivative, has attracted a lot of attention in conjugate construction because it has several meritorious characteristics such as full water solubility, tunable degradability, and modifiable functionalities. ${ }^{9,14-16}$ In particular, HES has been used as a plasma volume expander for years, making it notably advantageous over many other polymers for functioning as the conjugate backbone because of its high daily parenteral tolerance dose and good in vivo safety. ${ }^{4,15}$ Several HES-based anticancer conjugates have already been developed, and they exhibit varying degrees of improvements in anticancer efficacy compared to their respective corresponding free drugs. ${ }^{9,17-21}$

In the case of conjugate-based chemotherapy for solid tumor, many studies have revealed that many tumor cells often receive only a low or sublethal drug dosage due to the low intratumoral drug accumulation, poor cellular internalization, slow or insufficient intracellular drug release as well as heterogeneous drug distribution inside the tumor. As a result, these sublethally treated tumor cells are hardly eradicated. ${ }^{22-}$ ${ }^{24}$ Such insufficient chemotherapy has been considered as one of the important reasons for the tumor cell repopulation, the induced drug resistance, and tumor metastasis. ${ }^{23-25}$ Nowadays, it has been widely realized that a desirable conjugate should maintain the linker stable in the bloodstream and allow fast and sufficient drug release after its accumulation in tumor sites and internalization by tumor cells. ${ }^{4,10,26,27}$ In response to the challenges in conjugate-involved sublethal or insufficient chemotherapy, certain types of responsive bonds have been employed to modify the linkers of 
conjugates for achieving tumor-orientated drug accumulation together with fast and sufficient drug release. ${ }^{4,10,28,29}$

Disulfide bonds have been utilized to bridge the drug and the polymer backbone for building conjugates as they can be cleaved by glutathione (GSH) via a thiol-disulfide exchange reaction. ${ }^{19,30,31}$ It is known that there is a big difference between the intracellular GSH concentration $(2-10 \mathrm{mM})$ and the extracellular GSH concentration $(\sim 2$ $\mu \mathrm{M})$; and particularly, the intracellular GSH level of the tumor cells is at least four folds higher than that in the normal cells. ${ }^{30-33}$ Accordingly, a conjugate with a disulfide bond-bridged linker would be stable in the extracellular environment and release the loaded drug rapidly once endocytosed by the tumor cells.

$\mathrm{pH}$-responsive bonds have also been widely employed as a trigger for mediating the drug release from conjugates because $\mathrm{pH}$ values in some tissues or cellular compartments alter remarkably. ${ }^{34}$ The tumor matrix is known to be weakly acidic ( $\sim \mathrm{pH} 6.5)$ when comparing to the blood and normal tissues ( $\sim \mathrm{pH} 7.4)$, and the $\mathrm{pH}$ values of endosome and lysosome are in an even lower $\mathrm{pH}$ range between 5.0 and 5.5. ${ }^{35,36}$ Accordingly, a conjugate having $\mathrm{pH}$-responsiveness could deliver drugs towards tumors during its in vivo circulation since the $\mathrm{pH}$-sensitivity has been used for tumortargeting drug delivery. ${ }^{37-41}$ Among various kinds of $\mathrm{pH}$ sensitive bonds, the hydrazine bond has been used to trigger fast drug release from different nanocarriers because it can be rapidly cleaved in an acidic environment via hydrolysis. ${ }^{34,35}$ Considering the different responses of disulfide and hydrazone bonds, the antitumor efficacy of a conjugate with the linkage containing both disulfide and hydrazone bonds could be significantly enhanced since the disulfide and hydrazone bonds would synergistically trigger the rapid and sufficient drug release after conjugate initialization by tumor cells.

Toward this aim, a type of doxorubicin(DOX)-bound HES conjugate (HES-SS-hyd-DOX), for the first time, was synthesized using HES as a carrier while connecting DOX onto HES through a linker containing both disulfide and hydrazone bonds (Scheme 1). Meanwhile, hydrazone bond-bridged HES-hyd-DOX, disulfide bond-bridged HES-SS-DOX and unresponsive HES-DOX conjugates were also synthesized and used as control. Disulfide and hydrazone bonds in the linkage of the HES-SS-hyd-DOX conjugate were arranged in series so that DOX was bound by the hydrazone bond and
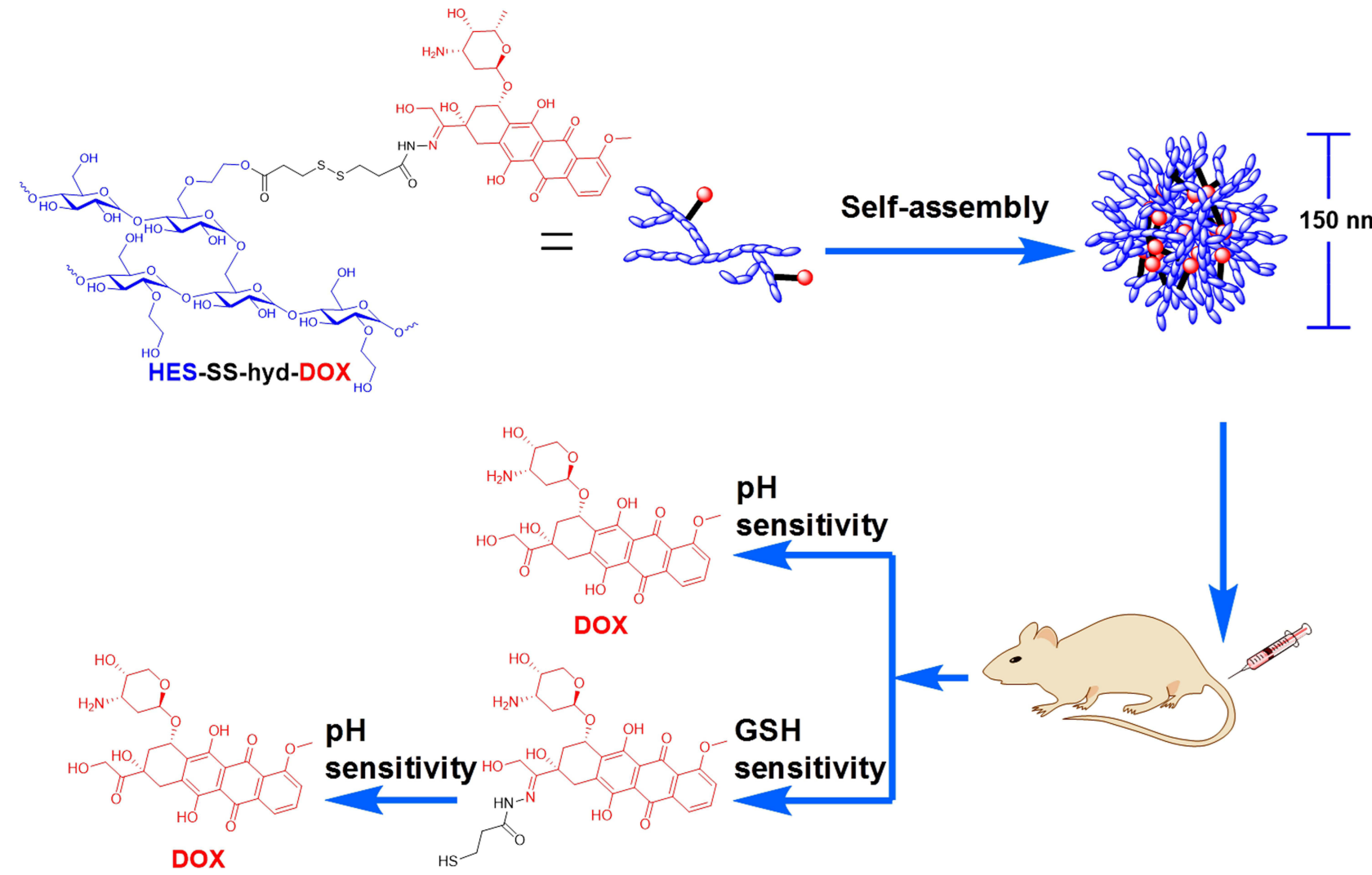

Scheme I Schematic illustrations for synthesis and self-assembly of HES-SS-hyd-DOX nanoparticles, and for mechanism of pH/redox responsive drug release from HES-SShyd-DOX nanoparticles. 
situated at the end of the linker, which will offer a prominent advantage of releasing DOX itself instead of its derivatives. A simple illustration was shown in the graphical abstract. Based on the advanced tumor model, the optimally obtained HES-SS-hyd-DOX NPs showed remarkably enhanced antitumor efficacy compared to HES-SS-DOX, HES-hyd-DOX, HES-DOX, and free DOX, respectively. The achieved results demonstrate that this novel type of HES-SS-hyd-DOX NPs has potential in clinical cancer chemotherapy in terms of its high anticancer efficacy and adequate safety.

\section{Experimental Section}

\section{Materials}

HES (Mw:130 kDa, hydroxyethyl molar substitution degree: 0.4) was procured from Wuhan HUST life Science \& Technology Ltd. (Wuhan, China). 3,3'-dithiodipropionic acid (DTDPA), 2,2'-dithiodipyridine (Py-ss-Py), octanedioic acid (ODA), 4-nitrophenyl chloroformate, hydrazine hydrate, methyl 3-mercaptopropionate, 3-mercaptopropionic acid (MPA), N-ethyl-N'-(3-(dimethylamino)propyl) carbodiimide hydrochloride (EDCI), DL-dithiothreitol (DTT), N-hydroxysuccinimide (NHS), 4-dimethylaminopyridine (DMAP), dicyclohexyl carbodiimide (DCC), and DOX were purchased from Aladdin Inc (Shanghai, China). Cyanine 5.5 mono NHS ester (Cy5.5-NHS) was purchased from Lumiprobe Corporation (Hunt Valley, USA). All other chemicals were of analytical grade and purchased from Sinopharm Inc, China.

\section{Synthesis and Characterization of HES-SS-hyd-DOX}

Four kinds of intermediates, 3-(2-pyridyldithio) propionic acid (PDP), HES-PDP, 3-mercaptopropanehydrazide (MPhyd), and HES-SS-hyd, were first synthesized via designed synthesis routes, as illustrated in Scheme 2. Details for their synthesis were provided in the Supplementary Materials. NMR measurements were used to identify the chemical structure of these intermediates, and their respective corresponding NMR spectra (Figure 1A and Figure S1-S3) were also presented in the Supplementary Materials.

The HES-SS-hyd-DOX conjugate was synthesized by capping DOX to the end of the side chains of HES-SS-hyd. In a typical synthesis procedure, a HES-SS-hyd solution was first prepared by dissolving $400 \mathrm{mg}$ of HES-SS-hyd in $10 \mathrm{~mL}$ anhydrous dimethyl sulfoxide. To this solution, $\mathrm{CF}_{3} \mathrm{COOH}(10 \mu \mathrm{L})$ and DOX (163.0 mg, $\left.0.28 \mathrm{mmol}\right)$ were added. The resulting mixture was vigorously stirred at 35 ${ }^{\circ} \mathrm{C}$ for $72 \mathrm{~h}$ in a nitrogen atmosphere. The mixture was subsequently added to a mixed solution (ethanol/ethyl ether $=1 / 1, \mathrm{v} / \mathrm{v}$ ) to produce a precipitate. The precipitate was collected by centrifugation at $8000 \mathrm{rpm}$ for $10 \mathrm{~min}$, and then, redissolved in dimethyl sulfoxide to prepare a new solution. This solution was introduced into a membrane tube (MWCO, 3500) and dialyzed against ultrapure water for 3 days. After freeze-drying, the achieved HES-SS-hydDOX product was stored at $4{ }^{\circ} \mathrm{C}$ for further use. The

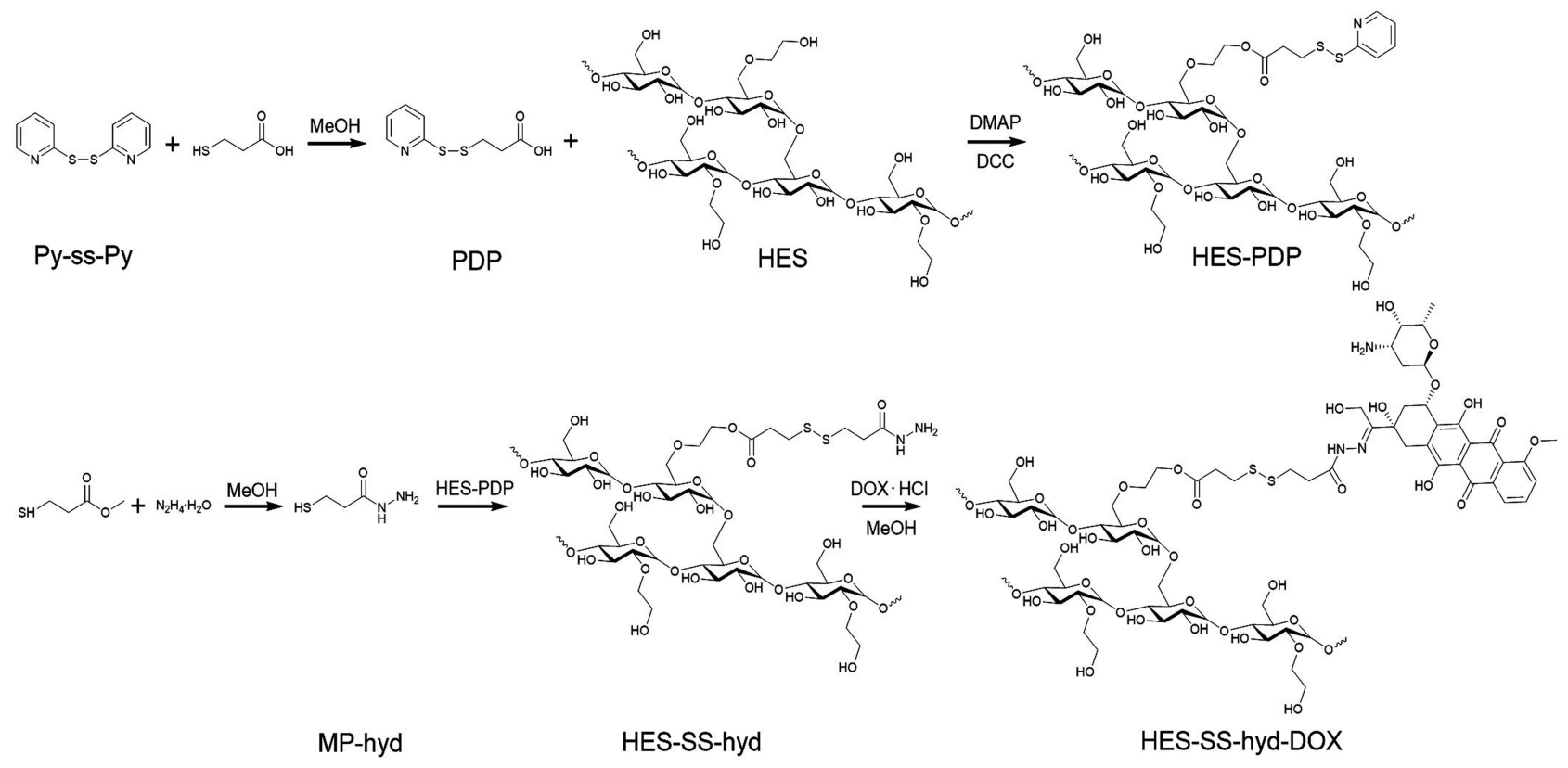

Scheme 2 Schematic illustration showing synthetic routes for four kinds of intermediates (PDP, HES-PDP, MP-hyd and HES-SS-hyd) and HES-SS-hyd-DOX conjugate. 
A

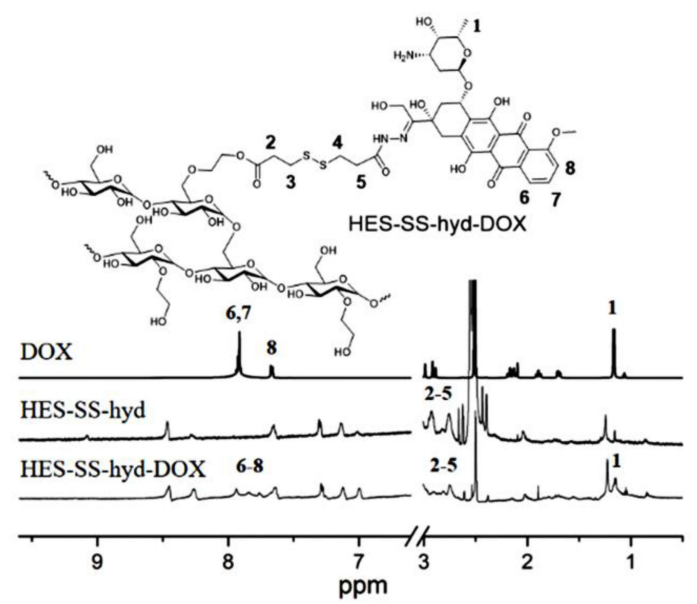

B

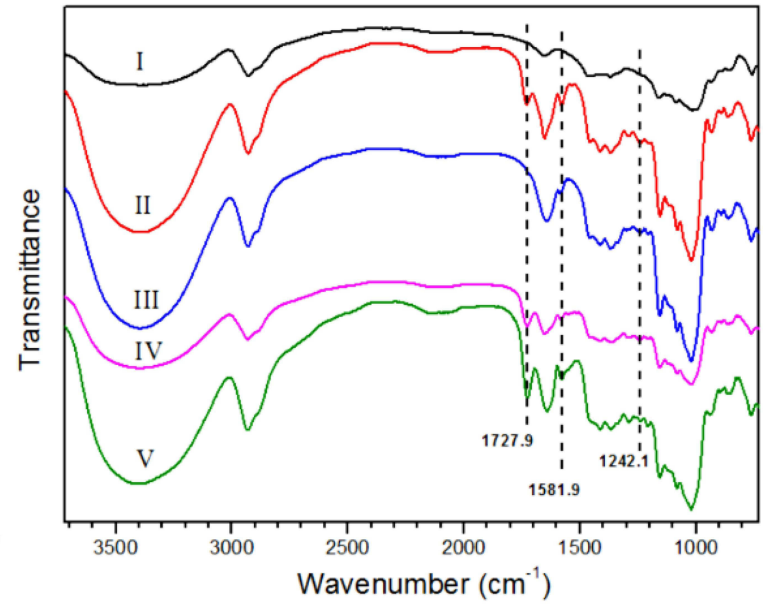

C

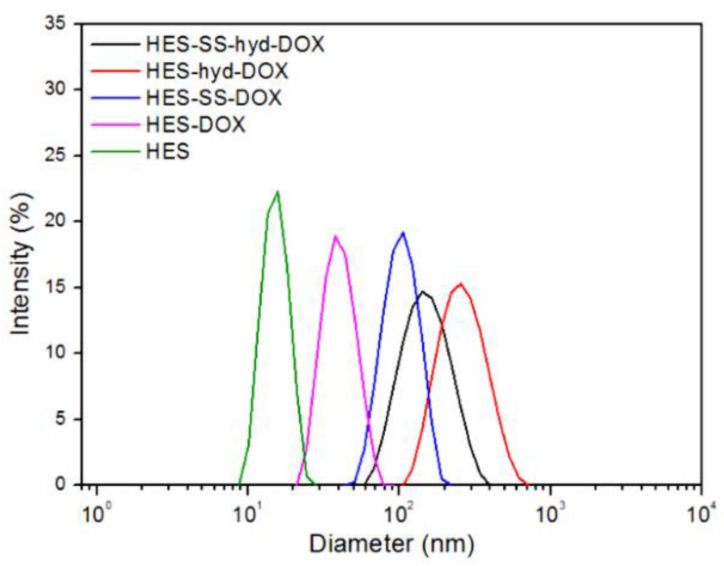

E

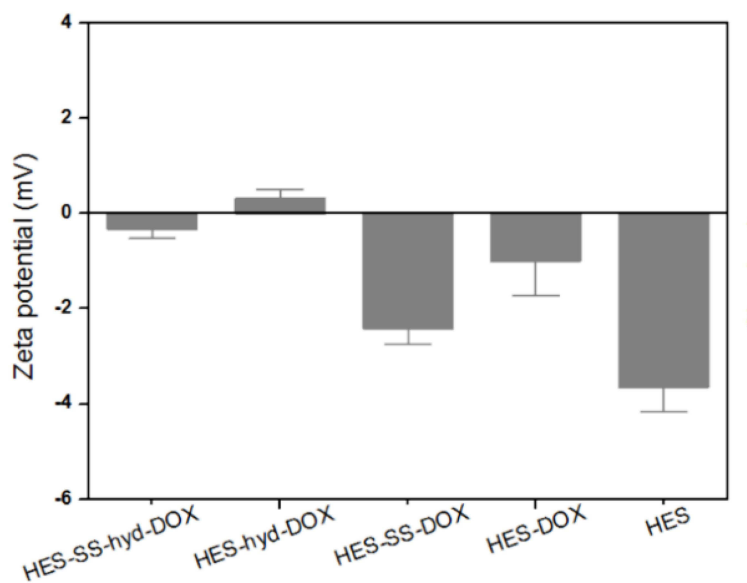

D

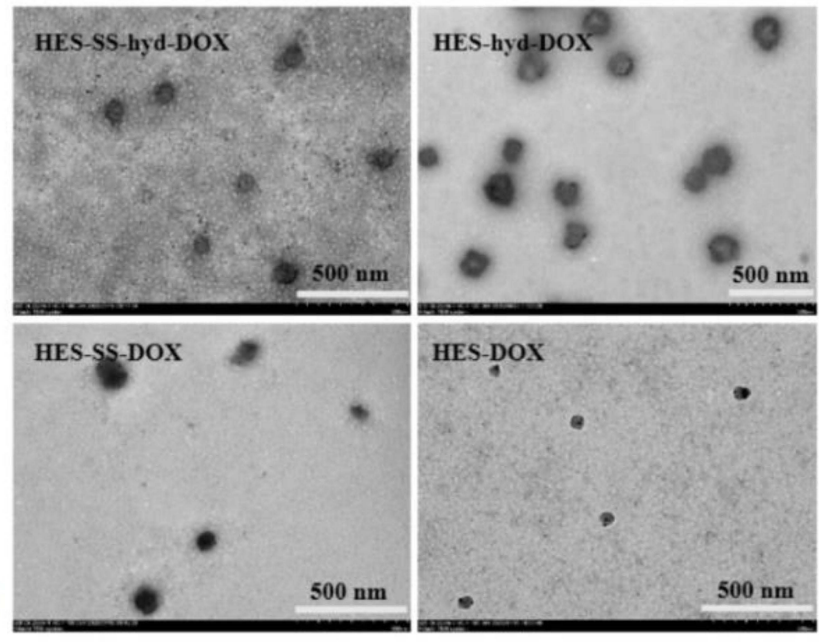

Figure I Characterization of the four kinds of conjugate NPs. (A) 'H NMR spectra of DOX, HES-SS-hyd and HES-SS-hyd-DOX. (B) FTIR spectra of HES (I), HES-DOX (II), HES-SS-DOX (III), HES-hyd-DOX (IV), and HES-SS-hyd-DOX (V). (C) Size distribution of HES and different conjugate NPs. (D) TEM images of different conjugate NPs. (E) Zeta potential for HES, HES-DOX, HES-SS-DOX, HES-hyd-DOX, and HES-SS-hyd-DOX NPs. (F) Size change of HES-SS-hyd-DOX NPs stored in PBS for various durations. 
structure of HES-SS-hyd-DOX was identified using NMR spectroscopy and the matched ${ }^{1} \mathrm{H}$ NMR spectrum was shown in Figure 1A.

\section{Synthesis of HES-hyd-DOX, HES-SS-DOX and HES-DOX}

Several conjugates with a single responsive bond (HES-SS -DOX and HES-hyd-DOX) or without any responsive bond (HES-DOX) were also synthesized and used as control. The synthesis routes (Scheme S1A and $\underline{\mathrm{S}}$ ) and detailed synthesis procedures for these conjugates together with their respective ${ }^{1} \mathrm{H}$ NMR spectra (Figure S4-S6) were also provided in the Supplementary Materials.

\section{Synthesis of HES-SS-hyd-Cy5.5}

Although DOX has a fluorescent property, its fluorescence imaging is usually interfered with by the strong background signal when it is used in vivo. ${ }^{42}$ Therefore, Cy5.5, a near-infrared fluorescent dye, was used to substitute DOX in the HES-SS-hyd-DOX conjugate for synthesizing a fluorescent counterpart, referred to as HESSS-hyd-Cy5.5. The resulting HES-SS-hyd-Cy5.5 NPs were used for imaging in vivo accumulation of NPs in the follow-up animal experiments.

HES-SS-hyd-Cy5.5 was synthesized as follows. The pre-synthesized HES-SS-hyd (300 mg) was dissolved in anhydrous dimethyl sulfoxide $(10 \mathrm{~mL})$ to prepare a HESSS-hyd solution. To this solution, DMAP $(5 \mathrm{mg})$ and a Cy5.5-NHS ester solution in DMSO $(5 \mathrm{mg} / \mathrm{mL}, 50 \mu \mathrm{L})$ were added. The resulting mixture was vigorously stirred at ambient temperature for $72 \mathrm{~h}$. Afterward, the mixture was processed through dialysis against ultrapure water for 3 days using a membrane tube (MWCO, 3500), followed by freeze-drying. The synthesis route for HES-SS-hydCy5.5 was illustrated in Scheme S1B in the Supporting Materials.

\section{Measurements}

${ }^{1} \mathrm{H}$ NMR spectra of different samples were recorded on a nuclear magnetic resonance spectrometer (AscendTM $600 \mathrm{MHz}$, Bruker) using $\mathrm{CDCl}_{3}$ or DMSO-d6 as solvents, and tetramethylsilane as an internal reference. FT-IR spectra were acquired using a Fourier transform infrared (FTIR) spectrometer (Vertex 70, Bruker) equipped with an attenuated total reflection accessory. A fluorescence spectrophotometer (F-4500, Hitachi) was employed for detecting fluorescence spectra of fluorescent samples.
Drug loading (DL) of conjugates was determined using a UV-vis spectrophotometer (TU-1901, Beijing Purkinje General Instrument Co., Ltd.) at an absorption wavelength of $481 \mathrm{~nm}$. The calibration curve was established using DOX/DMSO solutions with DOX concentration gradients. DL was calculated with the following formula:

$$
\mathrm{DL}(\%)=\frac{W 1}{W t} \times 100 \%
$$

Where $\mathrm{W} 1$ is the weight of the drug in the conjugate, and $\mathrm{Wt}$ is the weight of the conjugate.

\section{Self-Assembly of Conjugate Nanoparticles}

Four kinds of conjugates, HES-SS-hyd-DOX, HES-hydDOX, HES-SS-DOX, and HES-DOX, were tested to examine whether they could self-assemble into NPs in PBS. All these kinds of conjugates were processed into their respectively corresponding solutions with the same concentration of $2 \mathrm{mg} / \mathrm{mL}$. Each of the solutions was intermittently sonicated for $10 \mathrm{~min}$ at room temperature to facilitate the self-assembly of conjugates. The hydrodynamic size of the formed conjugate NPs was tested using a dynamic light scattering instrument (Nano-ZS90, Malvern); and the Zeta potential of NPs was also measured using the same instrument. The morphology of the resulting conjugate NPs was viewed using a transmission electron microscope (TEM, JEM-1230). Concerning TEM sample preparation, a drop of the conjugate suspension was placed onto a carbon-coated copper grid, stained with $2 \%$ phosphotungstic acid, and the samples were allowed to air-dry at room temperature overnight before TEM observation.

\section{In vitro Drug Release}

Release profiles for different conjugates, namely, HES-SShyd-DOX, HES-hyd-DOX, HES-SS-DOX, and HES-DOX, were determined using a dialysis method. Four kinds of solutions were employed as testing media to examine the drug release of conjugates at $37^{\circ} \mathrm{C}$ : (1) PBS buffer $(10 \mathrm{mM}$, pH 7.4); (2) PBS buffer (10 mM, pH 7.4) with DTT (10 $\mathrm{mM}$ ); (3) PBS buffer (10 mM, pH 5.0); and (4) PBS buffer $(10 \mathrm{mM}, \mathrm{pH} 5.0)$ with DTT $(10 \mathrm{mM})$. All these testing media contained a small amount of Tween-80 $(0.5 \%)$.

In a typical measurement process, $1 \mathrm{~mL}$ of the aqueous suspension of conjugate NPs was introduced into a membrane tube (MWCO, 3500), and the tube was then immersed in $30 \mathrm{~mL}$ of testing media in a capped flask at 
$37{ }^{\circ} \mathrm{C}$ with shaking at a speed of $100 \mathrm{rpm}$. At predetermined time points, $1 \mathrm{~mL}$ of medium was withdrawn and an equal volume of fresh media was replenished into the flask. The released DOX amount was determined via fluorescence measurement (F-4500, Hitachi) at $\lambda_{\mathrm{ex}}=501$ $\mathrm{nm}$ and $\lambda_{\mathrm{em}}=554 \mathrm{~nm}$, respectively.

\section{Cell Culture}

All kinds of cell lines used in this study were purchased from the Type Culture Collection of the Chinese Academy of Sciences (Shanghai, China). Murine hepatoma H22 cells were expanded using RPMI-1640 culture medium, supplemented with $10 \%$ fetal bovine serum, $100 \mathrm{U} / \mathrm{mL}$ penicillin, and $100 \mu \mathrm{g} / \mathrm{mL}$ streptomycin at $37{ }^{\circ} \mathrm{C}$ in a $5 \%$ $\mathrm{CO}_{2}$ atmosphere. Human hepatoma HepG2 cells were cultured under the same conditions, but in this case, the RPMI-1640 medium was replaced with the DMEM medium. Two types of expanded cells were suspended in PBS for further use.

\section{Tumor Model}

Six-week-old male BALB/c mice $(18.6 \pm 2.2 \mathrm{~g})$ were bought from the Hubei Provincial Center for Disease Control and Prevention (Wuhan, China) and used for animal experiments. They were housed in an air-conditioned atmosphere with a relative humidity of $50 \%$ under natural light/dark cycle conditions and allowed free access to standard food and water.

Tumor models were established by subcutaneously injecting $0.1 \mathrm{~mL}$ of $\mathrm{H} 22$ cell suspension $\left(2 \times 10^{6}\right.$ cells $\left./ \mathrm{mL}\right)$ to the right thigh of BALB/c mice. These cell-seeded mice were used for subsequent experiments when the induced tumors reached an estimated volume ranging between 170 and $190 \mathrm{~mm}^{3}$. Animal experiments were approved by the Animal Care and Use Committee of Huazhong University of Science and Technology (No. S915). All animal experiments were conducted following the NIH Guide for the Care and Use of Laboratory Animals.

\section{In vitro Cytotoxicity}

MTT assay was used to evaluate the in vitro cytotoxicity of conjugate NPs and free DOX against HepG2 cells. Briefly, HepG2 cells were seeded in 96-well plates at a cell density of $5 \times 10^{3}$ cells per well, and they were then treated with conjugate NPs and free DOX at various equivalent DOX dosages changing from $0.01 \mu \mathrm{g} / \mathrm{mL}$ to $10 \mu \mathrm{g} / \mathrm{mL}$, along with standard culture using the complete medium for $48 \mathrm{~h}$. 4 wells were set as duplications for each designated equivalent DOX concentration. After that, $20 \mu \mathrm{L}$ of MTT solution $(5 \mathrm{mg} / \mathrm{mL})$ was added into each well, followed by incubation for $4 \mathrm{~h}$. After evacuating the medium, DMSO $(150 \mu \mathrm{L})$ was added into each well for dissolving formazan crystals, and then, UV absorbance for each well was measured at an absorbance wavelength of $492 \mathrm{~nm}$ using a microplate reader (381C Microplate Reader). The group treated with a simple DMEM medium was used as a control, and the matched cell viability was considered as $100 \%$. The halfmaximum inhibition concentration $\left(\mathrm{IC}_{50}\right)$ was determined on the basis of cytotoxicity assessment.

\section{In vitro Cellular Uptake}

HepG2 cells were cultured with HES-SS-hyd-DOX, HEShyd-DOX, HES-SS-DOX, and HES-DOX NPs to assess their cellular uptake. In brief, the HepG2 cell suspension was introduced into confocal glass dishes to reach a volume of $1 \mathrm{~mL}$ at a cell density of $10^{5}$ cells $/ \mathrm{mL}$, and the cell-seeded dishes were incubated under standard conditions using the complete medium for $24 \mathrm{~h}$. Subsequently, cells in different dishes were treated with media containing either free DOX (control) or DOX-involved conjugate NPs (equivalent DOX dose: $5 \mu \mathrm{g} / \mathrm{mL}$ for all cell groups) for 4 $\mathrm{h}$ at $37^{\circ} \mathrm{C}$, respectively. The cells were then washed with PBS and fixed in a 4\% paraformaldehyde solution for 15 min. After that, $1.0 \mathrm{~mL}$ of 4.6-diamino-2-phenyl indole solution $(1 \mu \mathrm{g} / \mathrm{mL})$ was added to each dish to stain cells for $10 \mathrm{~min}$ before the confocal imaging (Leica TCS SP5).

\section{In vivo Imaging and Biodistribution}

HES-SS-hyd-Cy5.5 NPs were utilized to image the in vivo time-dependent accumulation of NPs. H22-tumor-bearing mice having an average tumor volume of around $190 \mathrm{~mm}^{3}$ were randomly divided into two groups with five mice in each group. Two groups of mice were intravenously administered with HES-SS-hyd-Cy5.5 NPs and free Cy5.5 (control), respectively. Whole-body fluorescence images of mice were recorded on a fluorescence imaging instrument (IVIS Lumina XR, Caliper), and images were taken starting from 15 min after the injection of HES-SS-hydCy5.5 NPs or free Cy5.5. During the period of imaging, mice were intermittently administered with a small amount of 3\% isoflurane anesthesia through a nose cone tube for continuous anesthesia when they were exposed to the camera.

Because of the fluorescence characteristics of DOX, the tumor-bearing mice were directly administered with 
free DOX or DOX-involved conjugate NPs, and their major organs were then excised for determining the ex vivo tissue distribution of DOX. Briefly, H22-tumorbearing mice were randomly divided into five groups, each group having five mice. Free DOX, HES-DOX, HESSS-DOX, HES-hyd-DOX, and HES-SS-hyd-DOX were dispersed in PBS to prepare five different solutions, and they were then applied to five groups of mice, respectively. Mice were injected with $0.1 \mathrm{~mL}$ of free DOX solution or DOX-loaded conjugate solution at an equivalent dosage of $4 \mathrm{mg} \mathrm{DOX} /(\mathrm{kg}$ of body weight) via the tail vein. At 12 $\mathrm{h}$ after injection, mice were sacrificed by cervical dislocation, and tumors as well as major organs (heart, liver, spleen, lung and kidney) were excised for the determination of fluorescence intensity using a fluorescence imaging instrument (IVIS Lumina XR, Caliper).

\section{In vivo Anti-Tumor Study}

H22-tumor-bearing mice were randomly assigned to six groups with four mice in each group and treated with five different DOX formulations for evaluating the antitumor efficacy of these drugs. Free DOX, HES-DOX, HES-SSDOX, HES-hyd-DOX, and HES-SS-hyd-DOX were intravenously administered to mice in five groups, respectively, at an equivalent DOX dose of $4 \mathrm{mg} /(\mathrm{kg}$ of body weight). The remaining group of mice was administered with PBS and used as control. The treatment was started when the tumor volume reached the range between 170 and $190 \mathrm{~mm}^{3}$. Injection for all mice was performed every 4 days 3 times in total, and the day for conducting the first injection was designated as day 0 .

The body weight of mice was measured every 2 days, and the tumor volume of mice was assessed using an empirical formula proposed in the literature. ${ }^{43}$ At the end of the treatment, mice were sacrificed, and tumors were excised for their weight determination and staining analysis. Major organs, including heart, liver, spleen, lung, and kidney, were also excised, fixed with paraformaldehyde, and sectioned into slices for the subsequent histopathological analysis using hematoxylin and eosin (H/E) staining. The images of H/E-stained tissue sections were examined by a pathologist.

\section{Statistical Analysis}

Statistical analyses were conducted with statistical software SPSS20. Statistical evaluations between two groups were compared using Student's $t$-test, and multiple comparisons were made using one-way analysis of variance.
Data were presented as mean \pm standard deviation, and the statistical difference was declared at $p<0.05$.

\section{Results and Discussion Synthesis of Conjugates}

Several kinds of HES products with different molecular weights and hydroxyethyl substitution degrees are now commercially available. ${ }^{15,44}$ Previous studies on HESbased NPs indicate that the molecular weight and hydroxyethyl substitution degree of HES are two key parameters for regulating the size and $\alpha$-amylase-mediated degradation of HES-based NPs. ${ }^{18,45,46}$ In this study, based on many comparative trials, HES with its molecular weight of $130 \mathrm{kDa}$ and hydroxyethyl molar substitution of 0.4 was selected as the carrier for DOX conjugation since it has a suitable ability to resist fast degradation, and concomitantly, the resulting NPs could have their sizes suited for intravenous administration and the tumor accumulation. A multi-step synthesis method, for the first time, was developed for synthesizing HES-SS-hyd-DOX to achieve a high DOX load, and meanwhile, to link DOX with HES through a hydrazone bond, as explicated in Scheme 2.

Two kinds of intermediates, PDP and HES-PDP, were first synthesized, and their ${ }^{1} \mathrm{H}$ NMR spectra were provided in

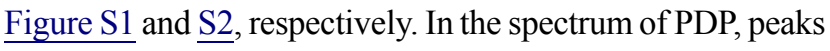
at 7.16, 7.61, 7.67, and $8.48 \mathrm{ppm}$ belong to the protons of pyridyl groups, and the peaks at 2.80 and 3.08 ppm are ascribed to the protons of methylene groups. HES-PDP was synthesized by conjugating PDP onto HES via an esterification reaction. In the spectrum for HES-PDP, the signals for the protons of pyridyl groups are registered in the range between 6.91 and $8.48 \mathrm{ppm}$, and the signals corresponding to the protons of methylene groups are seen between 2.92 and $3.23 \mathrm{ppm}$. Some other signals in the spectrum of HES-PDP are attributed to the HES backbone. ${ }^{45,46}$

The other two intermediates, MP-hyd and HES-SShyd, were also synthesized in advance, and their ${ }^{1} \mathrm{H}$ NMR spectra were presented in Figures $\mathrm{S} 3$ and $1 \mathrm{~A}$, respectively. In the ${ }^{1} \mathrm{H}$ NMR spectrum of MP-hyd, signals for the protons of hydrazide groups are observed at 4.18 and $9.00 \mathrm{ppm}$, whilst the signals for the protons of methylene groups can be seen at 2.27 and $2.66 \mathrm{ppm}$. Concerning the ${ }^{1} \mathrm{H}$ NMR spectrum of HES-SS-hyd, the proton signals of methylene groups in the range between 2.76 and 2.92 ppm demonstrate the successful reaction that occurred between MP-hyd and HES-PDP in terms of the formation of the disulfide bond. 
Based on the above-obtained intermediates, HES-SShyd-DOX was finally synthesized via a dehydration condensation reaction between HES-SS-hyd and DOX; and its ${ }^{1} \mathrm{H}$ NMR spectrum is presented in Figure 1A. The signals that appeared at $7.72-7.93 \mathrm{ppm}$ are assigned to the aromatic ring protons of DOX; the signals located $2.62-2.86 \mathrm{ppm}$ can be ascribed to the protons of methylene groups (- $\left.\mathrm{CH}_{2}-\mathrm{SS}-\right)$; and the signal at $1.35 \mathrm{ppm}$ should be attributed to the protons of methyl groups in DOX. FTIR spectrum for HES-SS-hyd-DOX is shown in Figure 1B. In comparison to HES, three new bands are seen to appear at around 1242,1581 , and $1727 \mathrm{~cm}^{-1}$ in the spectrum of HES-SS-hyd-DOX. The band at $1242 \mathrm{~cm}^{-1}$ is associated with the aromatic ring vibration of DOX, the band at $1581 \mathrm{~cm}^{-1}$ is assigned to the amide bonds closely connected with DOX, and the band registered at $1727 \mathrm{~cm}^{-1}$ is attributed to the stretching vibration of $\mathrm{C}=\mathrm{O}$ located in the linker between DOX and HES (see the inserted chemical structure of HES-SS-hyd-DOX in Figure 1A). Based on the results elucidated in Figure 1A and $\mathrm{B}$, it can be concluded that DOX has been successfully bound onto HES through a linker containing both disulfide and hydrazone bonds.

Several other conjugates, namely, HES-hyd-DOX, HES-SS-DOX, and HES-DOX, were synthesized and used for the comparison. The synthesis routes for them were respectively elucidated in Scheme S1A and $\underline{\mathrm{S} 2}$ in order. Chemical structures of these three kinds of conjugates were analyzed using NMR measurements and the matched ${ }^{1} \mathrm{H}$ NMR spectra with identified peaks for them are separately represented in Figure S4-S6. The FTIR spectra of these conjugates are shown in Figure 1B. It can be observed that HES-SS-DOX and HES-DOX have their FTIR spectra similar to that for HES-SS-hyd-DOX. These results are reasonable when three characteristic bands at 1242,1581 , and $1727 \mathrm{~cm}^{-1}$ are compared. HEShyd-DOX does not have the band at $1727 \mathrm{~cm}^{-1}$, which could be due to the presence of only a single carbonyl group in the linker (see chemical structure of HES-hydDOX in Figure S4. Results presented in Figure S4-S6 as well as in Figure 1B provide the collaborative evidence for the successful synthesis of HES-hyd-DOX, HES-SS-DOX, and HES-DOX. The DOX load in HES-SS-hyd-DOX, HES-hyd-DOX, HES-SS-DOX, and HES-DOX conjugates was determined using UV-vis spectrophotometry, and DL data calculated with the formula (1) for them are listed in Table 1.

\section{Parameters for Conjugate Nanoparticles}

Four kinds of conjugates, HES-SS-hyd-DOX, HES-hydDOX, HES-SS-DOX, and HES-DOX, were found to have amphiphilic characteristics due to the conjugation of hydrophobic DOX. Accordingly, they were further processed in aqueous media to test their self-assembly practicality with the help of sonication dispersion, and some results for the self-assembled NPs are shown in Figure 1. Considering the amphiphilic features of these conjugates, the resulting NPs would have a HES-exposed hydrophilic surface while encapsulating the bound DOX inside, as illustrated in Scheme 1. As a result, these NPs would have long in vivo circulation capability due to the high hydrophilicity of HES, and at the same time, be able to reduce the DOX-induced side effects because they are prone to accumulate in tumors via the EPR effect with dual responsive DOX release there.

Figure 1C shows representative hydrodynamic size distributions for the resulting NPs. These size distributions all exhibit approximate Gaussian distribution patterns with narrow distribution intervals. Several sets of samples for each kind of hydrated NPs were measured and data for the matched mean sizes are listed in Table 1. The size of drugload NPs is known to play an important role in regulating their drug release, cellular uptake, and in vivo biodistributions. ${ }^{13,47}$ Some studies have suggested that NPs with sizes in the range between 40 and $200 \mathrm{~nm}$ are more likely to accumulate in tumors via the EPR effect

Table I Parameters of Different Nanoparticles

\begin{tabular}{|l|l|l|l|l|}
\hline Sample Name & Mean Size (nm) & PDI $^{(\mathbf{a})}$ & $\zeta(\mathbf{m V})$ & DL (\%) \\
\hline HES-SS-hyd-DOX & $154.2 \pm 15.9$ & $0.294 \pm 0.0378$ & $-0.33 \pm 0.19$ & $5.15 \pm 0.16$ \\
HES-hyd-DOX & $267.5 \pm 22.3$ & $0.201 \pm 0.0144$ & $0.31 \pm 0.18$ & $4.94 \pm 0.29$ \\
HES-SS-DOX & $137.9 \pm 15.1$ & $0.394 \pm 0.0474$ & $-2.41 \pm 0.34$ & $6.31 \pm 0.27$ \\
HES-DOX & $42.5 \pm 3.4$ & $0.295 \pm 0.0211$ & $-1.00 \pm 0.73$ & $6.58 \pm 0.15$ \\
HES & $16.3 \pm 0.9$ & $0.145 \pm 0.0569$ & $-3.65 \pm 0.51$ & - \\
\hline
\end{tabular}

Notes: ${ }^{(a)}$ polydispersity index 
compared to others outside this size range. ${ }^{47,48}$ Table 1 shows that HES-SS-hyd-DOX NPs have their mean size of about $150 \mathrm{~nm}$, indicating that the HES-SS-hyd-DOX conjugate has been cleverly designed and synthesized so that it can be self-assembled into NPs with a befitting mean size.

TEM images in Figure 1D exhibit that four kinds of NPs were spherical and had good dispersibility. Figure 1E shows that the absolute value for the Zeta potential of these NPs was small, and HES-SS-hyd-DOX NPs had a nearly neutral surface charge nature. It is known that the physical stability of NPs is an important issue to their performance, ${ }^{12,48}$ HES-SS-hyd-DOX NPs were thus stored in PBS at $25{ }^{\circ} \mathrm{C}$ for 1 week to test whether their size significantly changed with storage time. No precipitation and coagulation were viewed for the stored HES-SS-hydDOX NPs during 7-day storage based on visual observations. Data in Figure 1F signify that the size of the hydrated HES-SS-hyd-DOX NPs changed slightly without significant differences and their PDI also displayed insignificant changes over 7 days, revealing that these HES-SShyd-DOX NPs are stable in PBS. Several other kinds of NPs were also tested to see their size change in PBS, and relevant data are depicted in Figure S7A. It can be noted that the curves present that HES-SS-DOX, HES-hydDOX, and HES-DOX NPs have insignificant size changes during 7-day storage in PBS, indicative of their welldefined stability.

In the following text, when referring to HES-SS-hydDOX, HES-SS-DOX, HES-hyd-DOX, and HES-DOX, they represent either their respective conjugates or selfassembled NPs, unless otherwise stated.

\section{In vitro Drug Release}

To determine the release patterns of HES-SS-hyd-DOX, HES-hyd-DOX, HES-SS-DOX, and HES-DOX, their respective suspensions in deionized water were used for release measurements. Each of the prepared suspensions was dialyzed against different PBS solutions with or without DTT as an analog of GSH while having varied $\mathrm{pH}$ values. As shown in Figure 2A, the release pattern of HES-SS-hyd-DOX behaved in dual parameter-dependent ways, DTT-dose and $\mathrm{pH}$ value. At a fixed $\mathrm{pH}$ value of 7.4, the DOX amount released from HES-SS-hyd-DOX sharply increased during the first 24-hour release period when the applied DTT dose was set as $0 \mathrm{mmol}$ and $10 \mathrm{mmol}$, respectively; and a similar situation occurred to these NPs when the $\mathrm{pH}$ value was fixed at 5.0 (Figure 2A). These results demonstrate that HES-SS-hyd-DOX NPs release the bound DOX in a dual responsive manner. In Figure $2 \mathrm{~B}$ and $\mathrm{C}$, it can be noticed that HES-hyd-DOX and HES-SS-DOX released their loaded DOX in a $\mathrm{pH}$ only or DTT-only responsive way. Regarding HES-DOX, their release patterns did not show significant responses (Figure 2D). In Figure 2D, the small difference caused by the $\mathrm{pH}$ change could be due to the partial hydrolysis of the DOX-bound linker in an acidic environment. Despite this small difference, by comparing the release patterns shown in Figure 2A and D in a paired manner, it can be drawn that after subtracting the small difference between them, the $\mathrm{pH}$-responsive release patterns illustrated in Figure 2A is still very significant, confirming that the $\mathrm{pH} / \mathrm{redox}$ responsive linkage in HES-SS-hyd-DOX has played a key role in regulating the release of the bound DOX. In this study, the hydrazone bond, disulfide bond and DOX in HES-SS-hyd-DOX were connected in a specifically designed order so that the final released product is DOX itself instead of its derivatives, as elucidated in Scheme 1.

\section{Cellular Uptake and in vitro Antitumor Activity}

Despite the fluorescent nature of DOX, its fluorescence would be self-quenched significantly given that DOX is bound onto HES due to the homo Förster resonance energy transfer. ${ }^{19}$ To verify this, the solution of free DOX and different conjugate suspensions with the same equivalent DOX concentration were subjected to the fluorescence measurement, and the obtained fluorescence spectra are presented in Figure S7B. All conjugates were seen to have the same characteristic peaks as that corresponding to free DOX but greatly reduced fluorescence intensity. These curves provide evidence for the fluorescence selfquenching of DOX after its conjugation onto HES. Nevertheless, the presently developed these DOX-bound conjugates were found to still be competent for use in testing the cellular DOX uptake in cancer cells. Figure 3A presents several sets of fluorescence images for HepG2 cells treated with free DOX or DOX-involved conjugates at an equivalent DOX dose of $5 \mu \mathrm{g} / \mathrm{mL}$ for $4 \mathrm{~h}$. It can be seen that the free DOX was localized both in the cytoplasm and the cell nucleus of HepG2 cells. For HESSS-hyd-DOX, the corresponding fluorescence images exhibit that the released DOX behaved in a way quite similar to free DOX while showing lower DOX fluorescence intensity compared to the free DOX. With regard to 
A

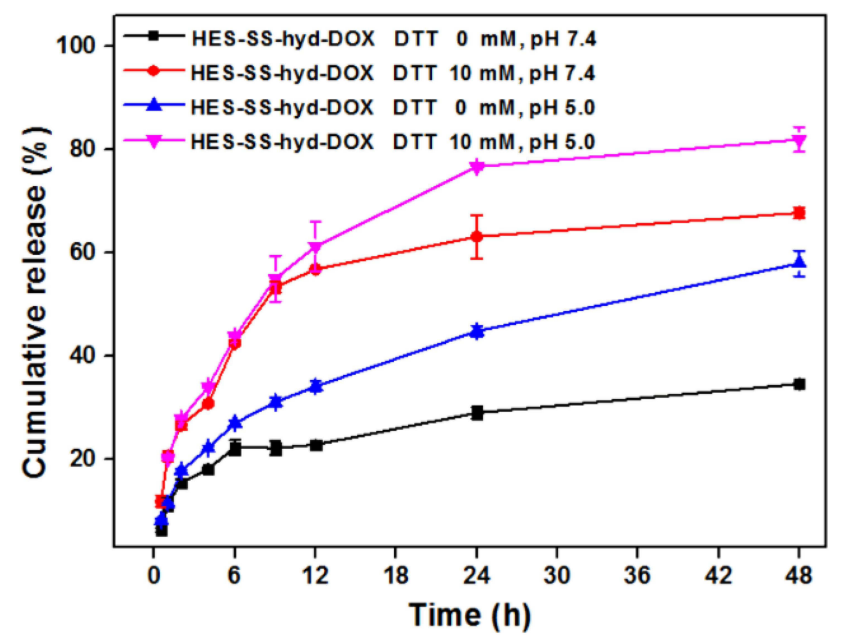

C

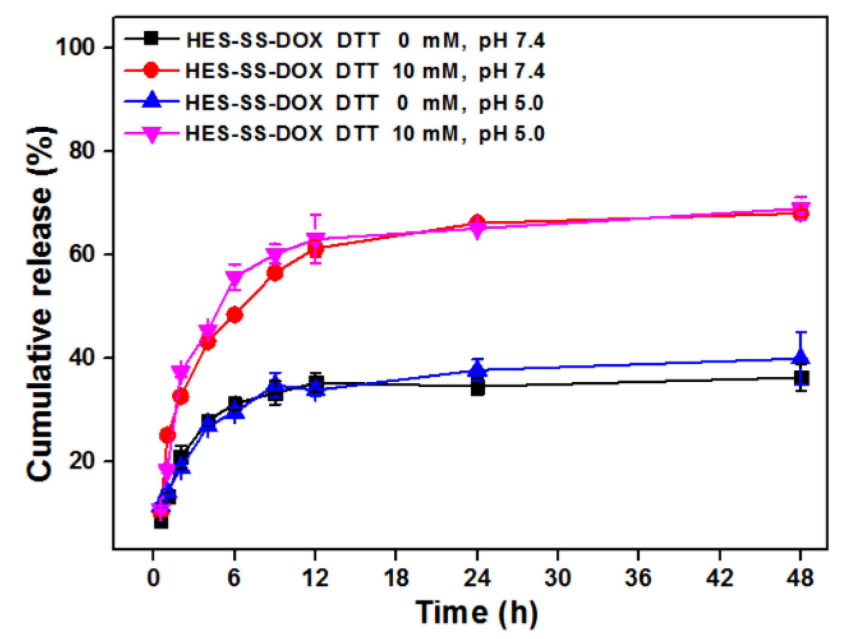

B

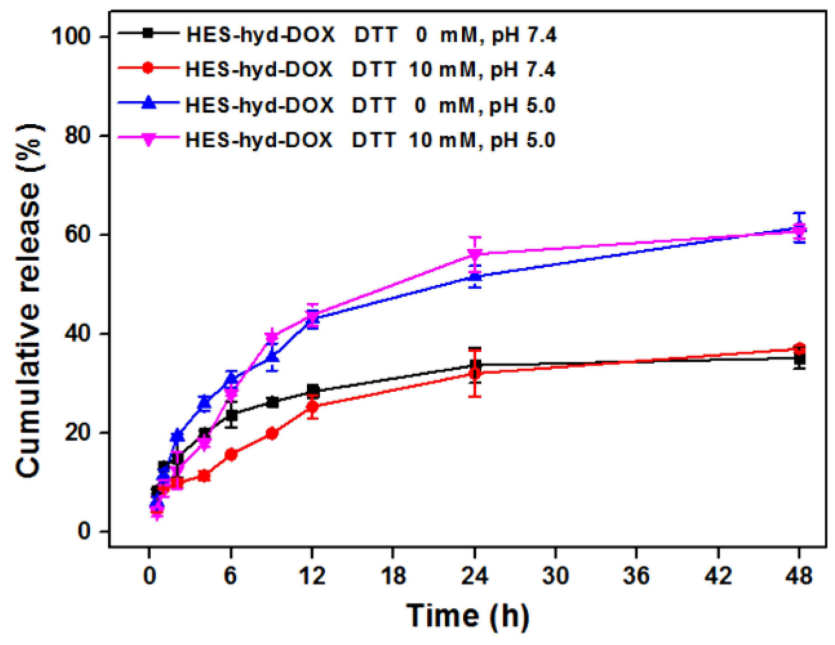

D

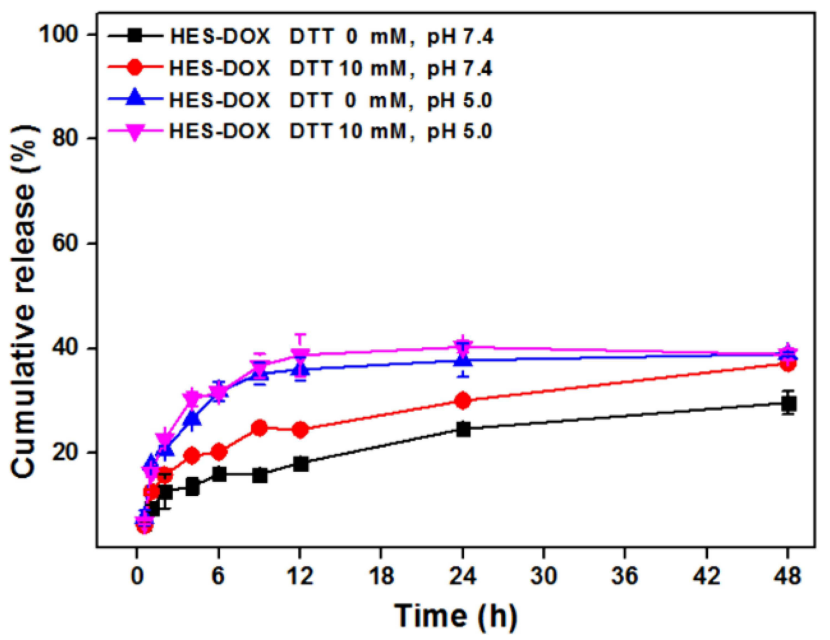

Figure 2 DOX release patterns of different NPs tested in PBS solutions with or without DTT as an analog of GSH while having varied $\mathrm{pH}$ values. (A) DOX release from HES-SS-hyd-DOX NPs. (B) DOX release from HES-hyd-DOX NPs. (C) DOX release from HES-SS-DOX NPs. (D) DOX release from HES-DOX NPs.

HES-hyd-DOX, HES-SS-DOX and HES-DOX, their respective DOX fluorescence intensities were pronouncedly lower than that for HES-SS-hyd-DOX. Considering a fact that DOX needs to play its role in the cell nucleus, the fluorescence intensity of the accumulated DOX in the nucleus of HepG2 cells would be an applicable indicator for assessing the DOX cellular uptake. Hence, the fluorescence intensity of DOX accumulated in the cell nucleus was detected and some results obtained in a semiquantitative manner are presented in Figure 3B. It can be noticed that the DOX fluorescence intensity corresponding to HES-SS-hyd-DOX group was much higher than that correlated to HES-hyd-DOX, HES-SS-DOX and HESDOX groups. The results explicated in Figure $3 \mathrm{~A}$ and B signify that compared to HES-hyd-DOX, HES-SS-
DOX and HES-DOX NPs, HES-SS-hyd-DOX NPs are more likely to be internalized by HepG2 cells, or release DOX faster or have both.

The cytotoxicity induced by free DOX and several kinds of conjugate NPs were tested to figure out whether the $\mathrm{pH} /$ redox responsive linkage in HES-SS-hyd-DOX is beneficial to its in vitro antitumor activity. HepG2 cells were used to assess the cytotoxicity of different DOX formulations, and the obtained data are graphed in Figure 3C. The bar graphs point out that free DOX, HESSS-hyd-DOX, HES-hyd-DOX, HES-SS-DOX, and HESDOX all show the ability to inhibit the growth of HepG2 cells in a dose-dependent manner. Among them, free DOX shows the highest growth inhibition effect on HepG2 cells. The high growth inhibition effect arisen from free DOX 
A

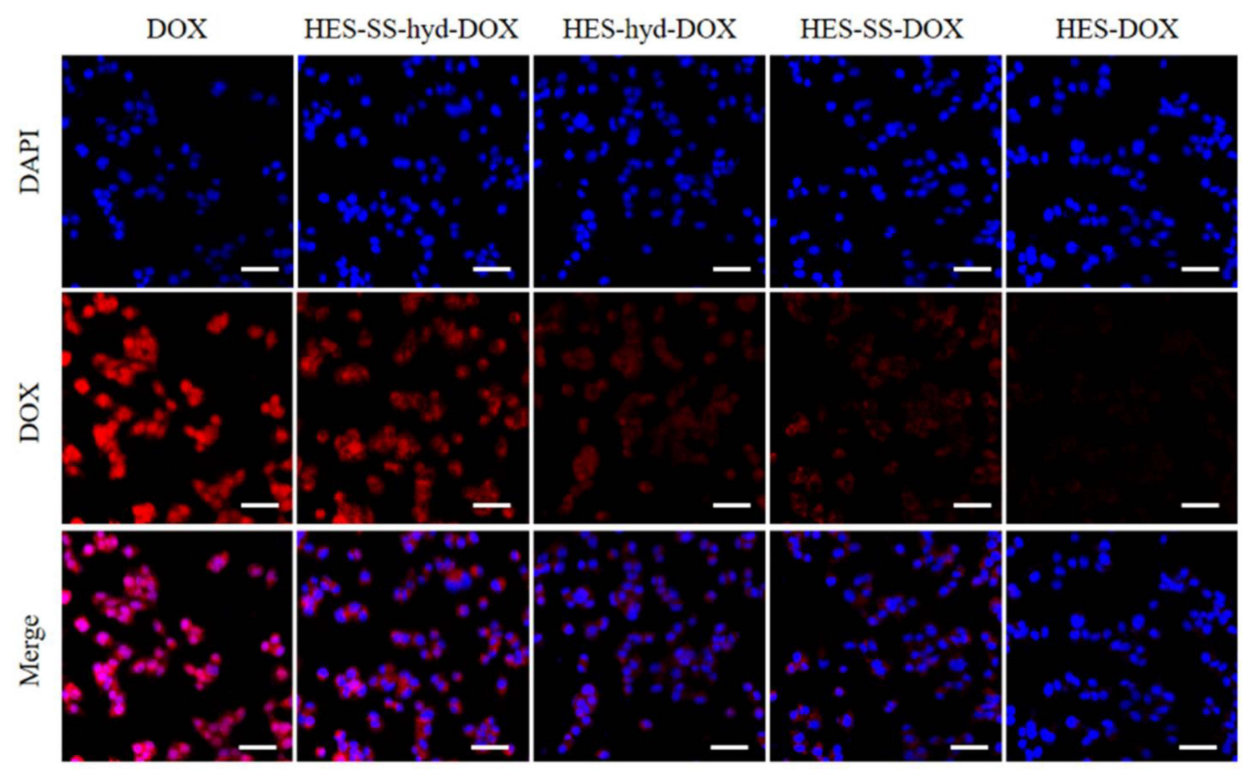

B

C
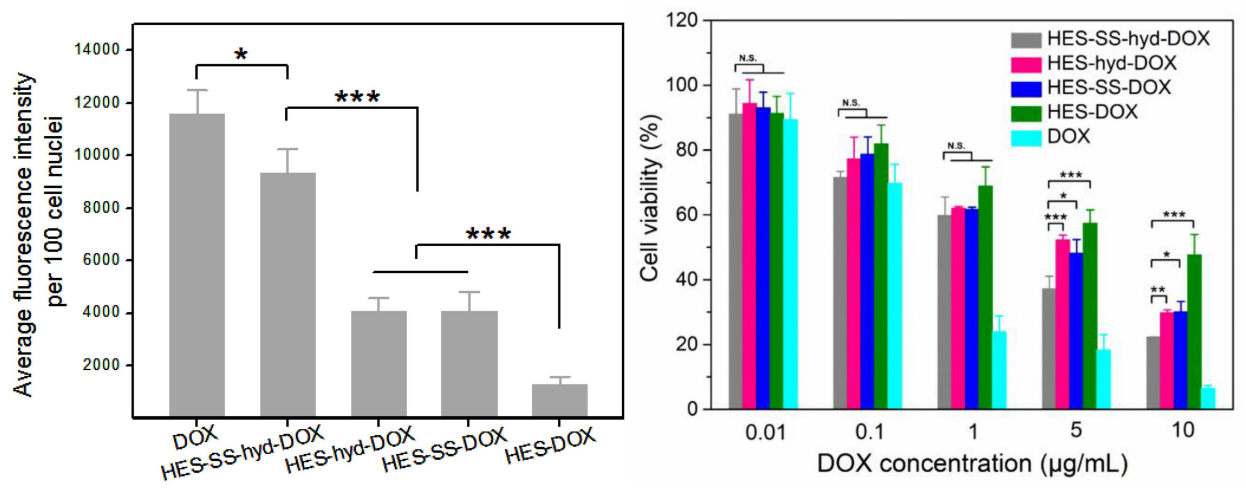

Figure 3 Cellular uptake and in vitro antitumor activity. (A) Fluorescence images of HepG2 cells treated by HES-SS-hyd-DOX, HES-hyd-DOX, HES-SS-DOX, HES-DOX NPs and free DOX (4h, equivalent DOX dose: $5 \mu \mathrm{g} / \mathrm{mL}$ ). (B) Average fluorescence intensity of DOX accumulated in the nuclei of HepG2 cells that were treated by HES-SShyd-DOX, HES-hyd-DOX, HES-SS-DOX, HES-DOX NPs and free DOX (4h, equivalent DOX dose: $5 \mu \mathrm{g} / \mathrm{mL}$ ). (C) The viability of HepG2 cells treated with various amounts of HES-SS-hyd-DOX, HES-hyd-DOX, HES-SS-DOX, HES-DOX and free DOX for $48 \mathrm{~h}(* p<0.05$; **p $<0.0 \mathrm{l}$; ***p $<0.00 \mathrm{I}$; N.S., no significance).

can be attributed to the fact that free DOX can be readily transported into cells by way of passive diffusion. About DOX conjugate NPs, their hydrophilic HES backbone hampers their internalization. ${ }^{49}$ Besides, DOX released from conjugate NPs is quite dependent on the timeconsuming disconnection of the linkage bridging between DOX and HES. These two factors jointly result in lower growth inhibition of these conjugate NPs against HepG2 cells when compared to free DOX. By comparing four kinds of conjugates with each other, it can be noticed that HES-SS-hyd-DOX has a significantly higher growth inhibition effect on HepG2 cells with their $\mathrm{IC}_{50}$ value of 2.22, 1.97, and 7.12 times lower than that for HES-hydDOX, HES-SS-DOX, and HES-DOX (Table S1), respectively. Accordingly, the higher growth inhibition effect of HES-SS-hyd-DOX on tumor cells can be ascribed to its $\mathrm{pH} /$ redox responsive linkage. After uptake by HepG2 cells, the linker in HES-SS-hyd-DOX would be disconnected due to the intracellular acidic environment and the high GSH level, resulting in fast and sufficient drug release and timely cytotoxicity.

\section{In vivo Imaging and Tissue Biodistribution}

HES-SS-hyd-Cy5.5 was synthesized following a synthesis route similar to that for the synthesis of HES-SS-hyd-DOX to substitute HES-SS-hyd-DOX for evaluating the conjugate accumulation in tumor since in vivo DOX fluorescence signal is usually interfered by a strong background 
fluorescence signal. ${ }^{42}$ The HES-SS-hyd-Cy5.5 conjugate had the same structural features when compared to HESSS-hyd-DOX and was also able to self-assemble into NPs in an aqueous environment. The resulting HES-SS-hydCy5.5 NPs were found to have their size-distribution and nearly neutral surface potential similar to that for HES-SShyd-DOX (Figure S8 and S9). In addition, HES-SS-hydCy5.5 was capable of maintaining its fluorescence intensity almost the same as that matched with free Cy5.5 dye without significant self-quenching (Figure S10). Therefore, HES-SS-hyd-Cy5.5 would be an appropriate analog to HES-SS-hyd-DOX for in vivo imaging.

Two sets of fluorescence images taken from the back of H22-tumor-bearing mice are displayed in Figure 4A. Images show that a very weak fluorescence signal appeared in the vicinity of the tumor region at $15 \mathrm{~min}$ after injection of free Cy5.5, and it reached the highest intensity after $2 \mathrm{~h}$ injection. Thereafter, the signal intensity dropped down sharply to almost disappear at around 12 $\mathrm{h}$ after injection, meaning that free Cy5.5 has only a short circulation life. In contrast to the images assigned to free Cy5.5, a bright fluorescence signal associated with HESSS-hyd-Cy5.5 NPs was shown in the tumor region at 15 min after their injection, and subsequently, the intensity of fluorescence signals remained at an almost similar level in the tumor area until $48 \mathrm{~h}$. These images demonstrate that HES-SS-hyd-Cy5.5 NPs have a much prolonged blood circulation life compared to free Cy5.5, and also show the tumor-oriented accumulation characteristics. Semiquantitative analysis results of the fluorescence intensity for tumor regions are represented in Figure 4B. The curve for HES-SS-hyd-Cy5.5 shows that the fluorescence intensity of tumor sites increased as time advanced, and reached the peak level at $1 \mathrm{~h}$ after injection; and thereafter, it slightly declined but was maintained at a high level up to $48 \mathrm{~h}$. The fluorescence intensity corresponding to free Cy5.5 was seen to be much lower than that for HES-SShyd-Cy5.5 over the whole sampling time range. Since free Cy5.5 (Cyanine 5.5 mono NHS ester) is a small molecule fluorescence dye, it will thus be easily cleared out of the bloodstream after its intravenous administration owing to its high hydrophobicity. In contrast, HES-SS-hyd-Cy5.5 NPs have a highly hydrophilic surface with a desirable mean size (Scheme 1, Figures 1 and $\underline{\mathrm{S} 8}$ ), which enables them to be effectively accumulated in tumors via the EPR effect. Figure $4 \mathrm{C}$ shows representative ex vivo images of main organs and tumors excised from mice administered with different DOX formulations for $48 \mathrm{~h}$, and the semi- quantitative fluorescence intensity detected from the excised organs and tumors is presented in Figure 4D. The images in Figure 4C display that DOX-bound NPs were accumulated in the liver and tumor, whereas free DOX was accumulated in the liver, kidney, and tumor. Bar graphs in Figure 4D indicate that the accumulated DOX amount released from conjugate NPs in the liver was similar to that of free DOX without a significant difference; but in tumor sites, the detected DOX amount corresponding to HES-SS-hyd-DOX was nearly 1.9 times higher than that of free DOX, HES-hyd-DOX, and HESDOX, and 1.4 times higher than that of HES-SS-DOX, respectively. The high tumor-accumulated DOX amount detected from HES-SS-hyd-DOX can be ascribed to that HES-SS-hyd-DOX tends to accumulate in the tumor (Figure 4A and B). In comparison to DOX-bound NPs, the higher free DOX accumulation in the kidney can be attributed to its low molecular weight, which results in its rapid clearance from blood circulation via renal elimination.

\section{In vivo Antitumor Efficacy}

H22-tumor-bearing mice were intravenously administered with different DOX formulations at an equivalent dose of $4 \mathrm{mg} \mathrm{DOX} /(\mathrm{kg}$ of body weight) to assess the antitumor efficacy of applied agents, and relevant results are elucidated in Figure 5A-E. Time-varying tumor volume curves reveal that all treated groups exhibited tumor growth inhibition compared to the control group, and HES-SS-hydDOX group had significantly stronger tumor growth inhibition in comparison to others. The images for excised tumors (Figure 5B) and the changes in tumor weight (Figure 5C) provided further evidence that tumor growth in the HES-SS-hyd-DOX group was more significantly inhibited than that in other groups. At the end of treatment, the tumor volume matched with the mice treated with HES-SS-hyd-DOX NPs was 1.43-, 1.64-, 1.85-, 2.31, and 3.41-fold lower, and meanwhile, the corresponding tumor weight was 1.39-, 1.79-, 1.98-, 2.56- and 3.19-fold lower when compared to those in HES-SS-DOX, free DOX, HES-hyd-DOX, HES-DOX and PBS (control) groups, respectively. The best in vivo antitumor efficacy of HES-SS-hyd-DOX can be attributed to its tumororiented accumulation, and rapid DOX release regulated by its $\mathrm{pH} /$ redox responsive linkage. The bodyweight of mice in all groups was measured for evaluating the toxicity possibly arisen from repeated administration of DOXinvolved agents, and relevant data are plotted in 
A
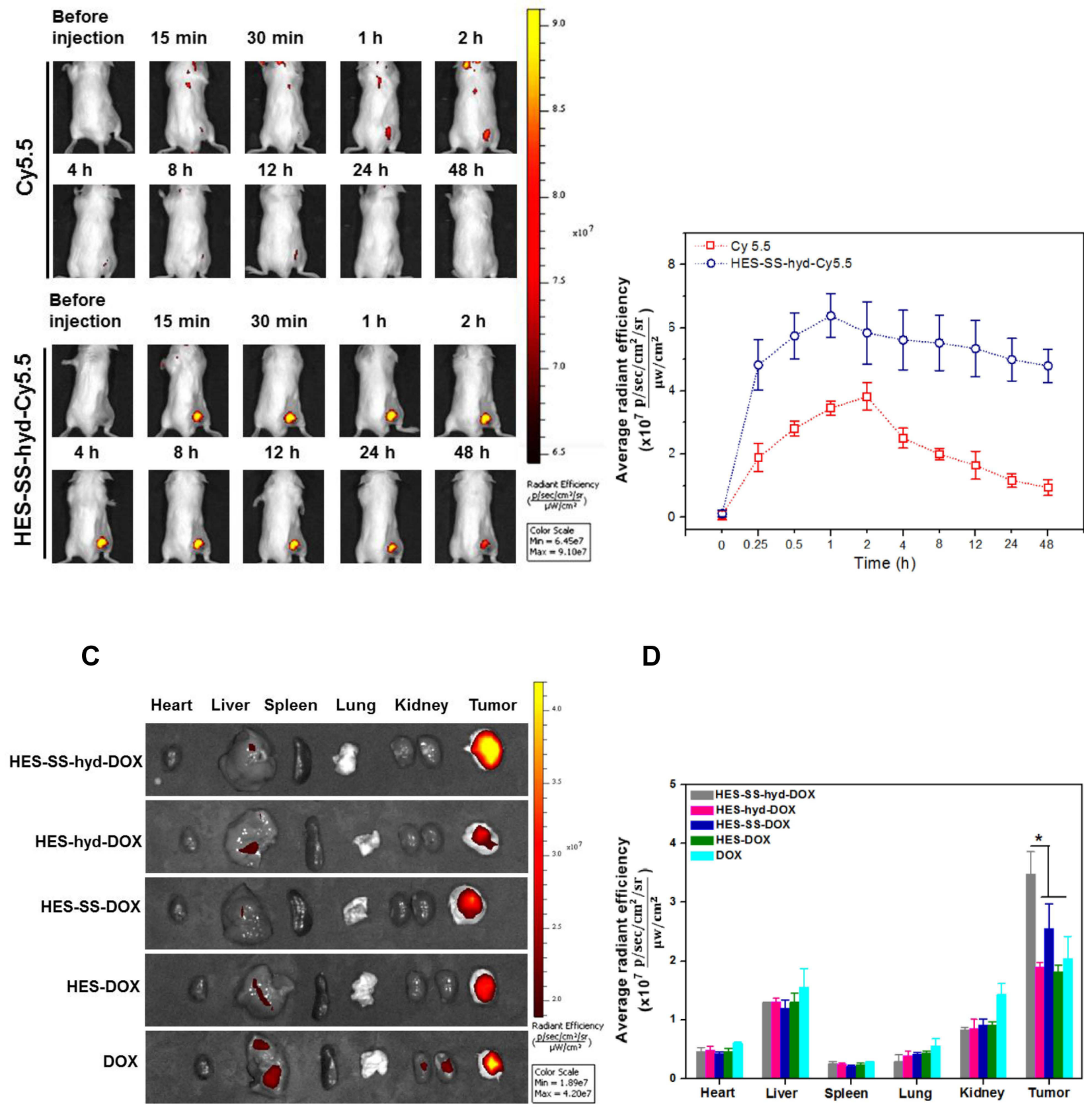

Figure 4 In vivo imaging and tissue biodistribution. (A) Representative real-time fluorescence images of H22-tumor-bearing mice administered with free Cy5.5 and HES-SShyd-Cy5.5 NPs (equivalent Cy5.5 dose: $50 \mu \mathrm{g} /(\mathrm{kg}$ of body weight)). (B) Average radiant efficacy of tumor site at different time points. (C) Fluorescence image of ex vivo organs and tumors. (D) Average radiant efficacy of major organs and tumors $(* p<0.05)$.

Figure 5D. Significant weight loss was detected from the mice in the free DOX group starting from around day 4 after the first injection, indicating DOX-induced toxic effects. As for the mice in other groups, their body weight increased to a certain extent at the end of the treatment, and there was no significant difference in the average body weight among these groups. The reduced side effects of conjugate NPs could be attributed to (I) their self- assembly by which DOX was encapsulated inside NPs; and (II) their tumor-oriented accumulation due to the EPR effect.

The tumors harvested at the end of the treatment were sectioned into slices for histological analysis using $\mathrm{H} / \mathrm{E}$ staining to further evaluate the effect of applied agents on tumors, and the obtained images are represented in Figure 5E. The mice in PBS groups were used as a control. By comparing 
A

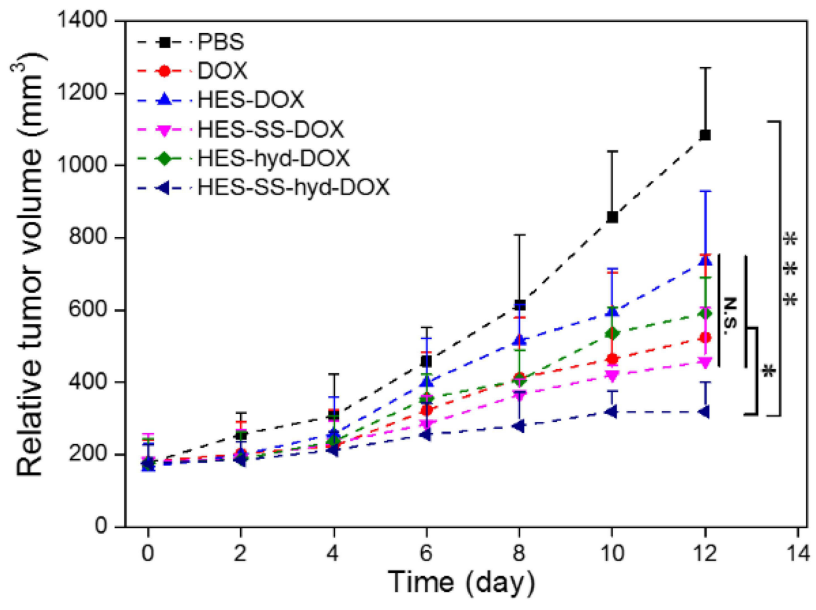

C

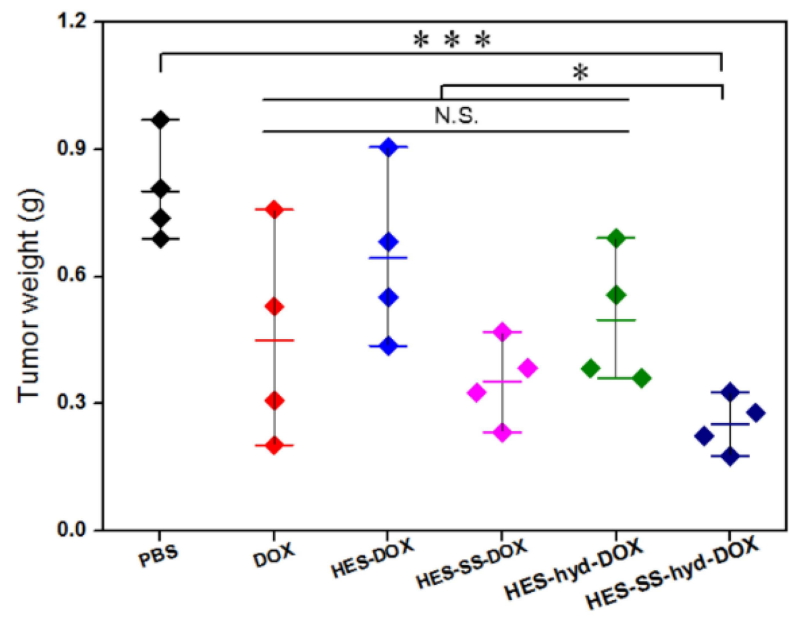

E

PBS

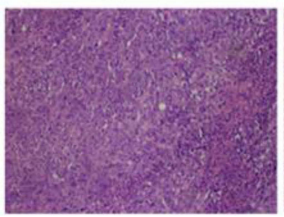

DOX

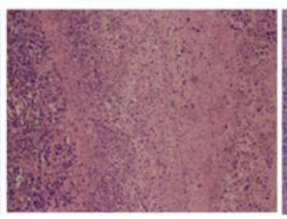

HES-DOX

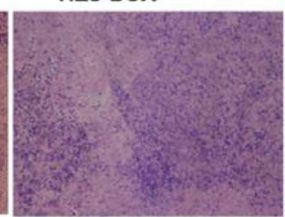

B

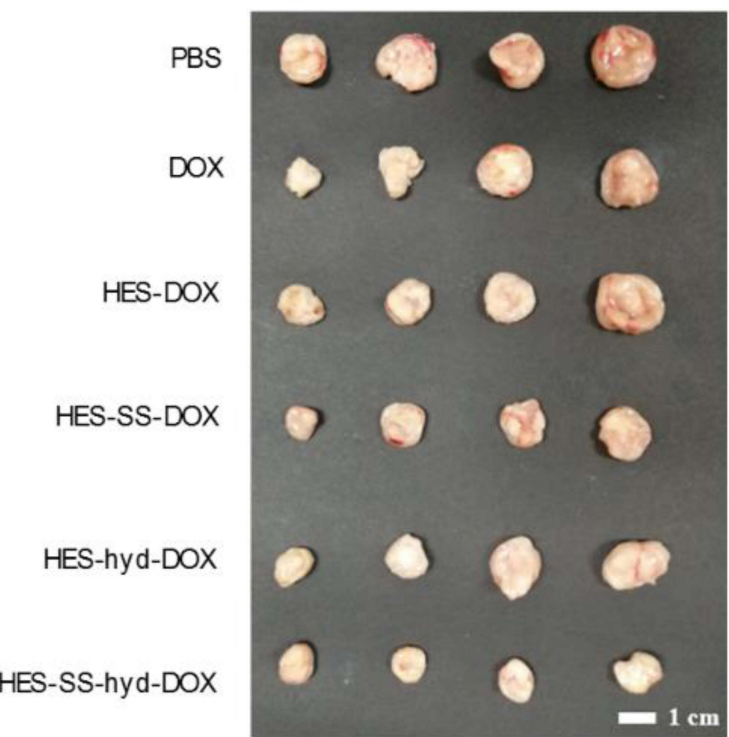

D

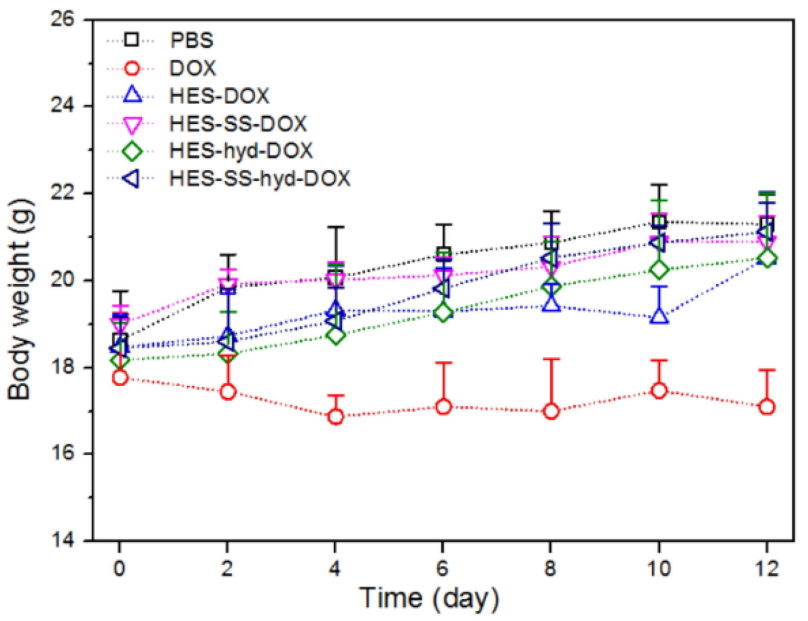

HES-SS-DOX

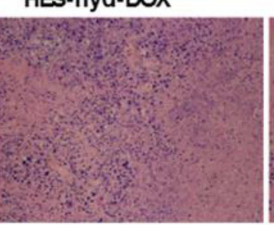

HES-SS-hyd-DOX
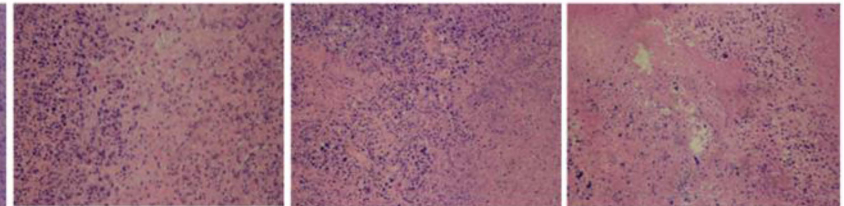

Figure 5 In vivo antitumor efficacy. (A) Time-varying tumor volumes during treatment. (B) Images of excised tumors after treatment. (C) Average weights of excised tumors after treatment. (D) Changes in body weight. (E) H/E staining micrographs for H22-tumor-bearing mice treated with different DOX formulations (equivalent DOX dose: $4 \mathrm{mg} /\left(\mathrm{kg}\right.$ of body weight); ${ }^{*} p<0.05$; ${ }^{* *} p<0.001$; magnification, 200x), N. S, no significance.

the dark purple color area among these micrographs, it can be reached that HES-SS-hyd-DOX had significantly higher cytotoxicity to the cancer cells, indicated by the large purple-free area. Two representative images with higher magnification for HES-SS-hyd-DOX and PBS groups are displayed in Figure S11. In comparison to the image for the PBS group, the image matched with the HES-SS-hyd-DOX group shows that most of its area did not have any stained 
purple at all; and in the small stained area, the cell nucleus appeared to be shrunken, irregular or broken, confirming that a large number of cells in the tumors belonging to HES-SShyd-DOX group have already been necrotic or apoptotic.

The significantly higher anti-cancer cytotoxicity of HES-SS-hyd-DOX can be attributed to its enhanced intratumoral accumulation (Figure 4C), and fast DOX release (Figure 2A). It is worth mentioning that the initial tumor volume before treatment in the current study was between $170-190 \mathrm{~mm}^{3}$, which is considerably larger than that mentioned in some previous studies, ${ }^{19,45,46,50}$ and can be considered as advanced tumor models. Therefore, the presently achieved results corroborate that these HES-SS-hyd-DOX NPs have significantly higher antitumor efficacy because they can inhibit the growth of advanced tumors.

DOX-induced cardiotoxicity is one of the major concerns related to its applications. ${ }^{51,52}$ Histological analyses were thus performed on major organs such as the heart, liver, spleen, lung, and kidney to evaluate the toxicity of different DOX Formulations. The tissue sections of mice in different groups were stained with $\mathrm{H} / \mathrm{E}$, and the obtained micrographs are presented in Figure 6 .
Micrographs obtained from the sections respectively belonging to HES-SS-hyd-DOX, HES-hyd-DOX, HESSS-DOX and HES-DOX groups exhibit that the stained tissues had normal histological structures when compared to the corresponding ones in the control group. Micrographs matched with the free DOX group show that the liver, spleen, lung, and kidney of the treated mice had normal tissue structures, but the cardiac tissue had some visual pathological changes, ${ }^{53,54}$ as indicated by the hypertrophy and orientation disorder of myocardium (white arrows), the vacuoles with varying shapes and sizes (yellow arrows), and the aggregated inflammatory cardiac muscle cells (ellipse marked by white dotted line). Results presented in Figure 6 reveal that these DOX-bound conjugate NPs have not resulted in impairments to the normal organs of the treated mice. The in vivo safety of conjugate NPs may come from the effects of their self-assembly. As shown earlier, HES-SS-hyd-DOX, HES-hyd-DOX, HESSS-DOX, and HES-DOX conjugates can self-assemble into NPs in aqueous media, which will greatly reduce DOX exposure in the circulation system on the one hand, and enhance DOX accumulation in the tumor with DOX release there on the other.

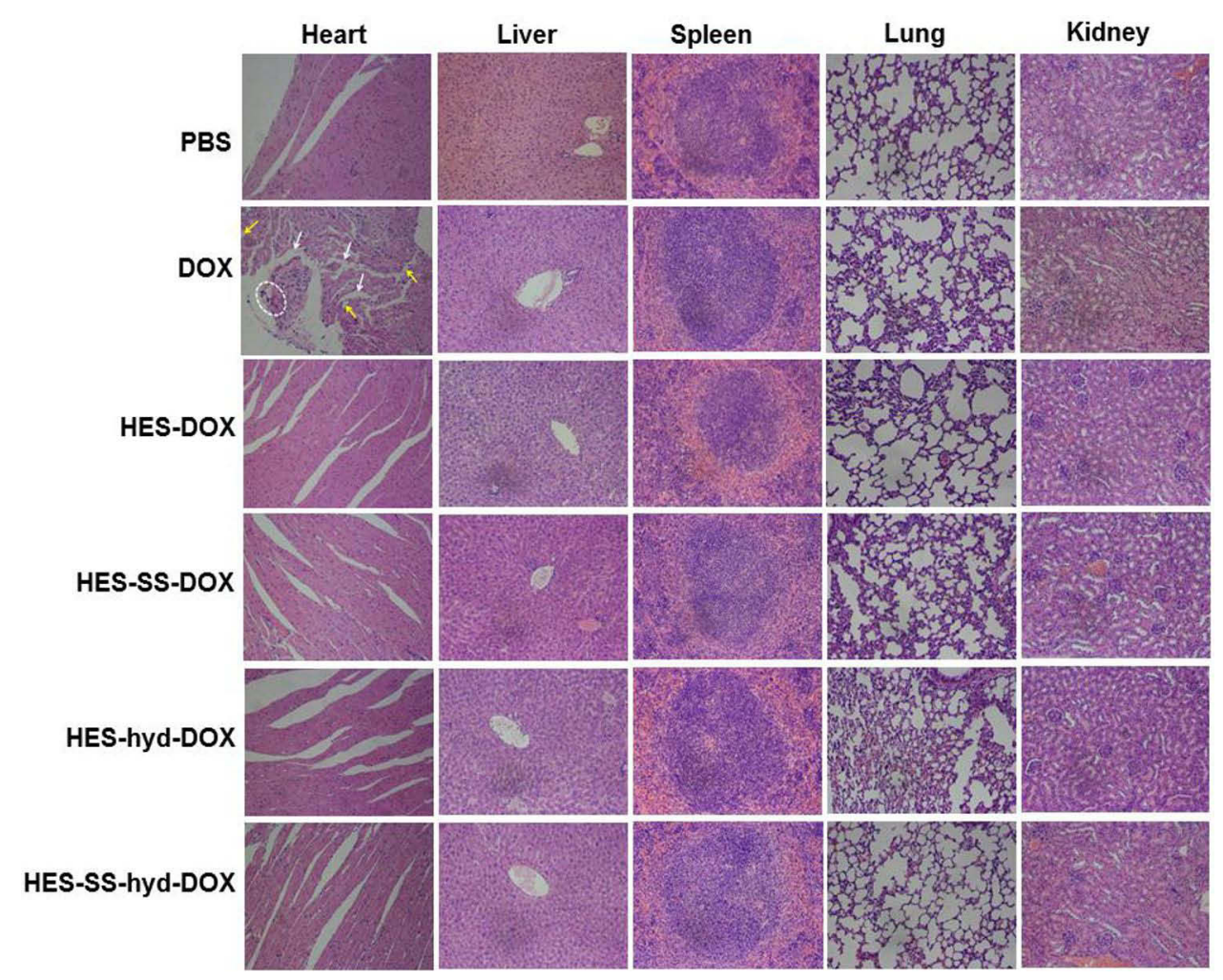

Figure 6 Representative micrographs of H/E-stained tissue sections respectively matching with major organs excised from $\mathrm{H} 22$-tumor-bearing mice treated with different DOX formulations (organs were harvested after 12-day treatment, equivalent DOX dose: $4 \mathrm{mg} /(\mathrm{kg}$ of body weight); magnification, $200 \times$ ). 


\section{Conclusions}

A novel type of DOX-bound HES conjugate NPs with designed structures and desirable properties was successfully developed. DOX was bound onto HES via a linker containing both disulfide bond and hydrazone bond in series to form a specific kind of conjugate (HES-SS-hydDOX) with characteristics for releasing of DOX itself instead of its derivatives. HES-SS-hyd-DOX was able to self-assemble into NPs in aqueous media with suitable sizes, allowing HES-SS-hyd-DOX NPs to circulate long in the bloodstream and to efficiently accumulate in the tumor. The $\mathrm{pH} / \mathrm{redox}$ responsive linkage in HES-SS-hydDOX was found to play a critical role in fast and sufficiently releasing the bound DOX so that the HES-SShyd-DOX-caused cellular DOX uptake in tumor cells was very similar to that of free DOX. In vivo results obtained from H22-tumor-bearing mice proved that HES-SS-hydDOX NPs were capable of inhibiting the growth of advanced tumors with significantly higher antitumor efficacy in comparison to free DOX, and to those DOX-bound NPs that have an only single responsive or unresponsive bond. In addition, HES-SS-hyd-DOX NPs showed almost no impairment to the main organs of the mice. Considering the fully biodegradable and biocompatible nature of HESSS-hyd-DOX NPs as well as their safe and high antitumor performance, they hold quite promising potential in translation for clinical trials.

\section{Acknowledgments}

This work was financially supported by the National Key R\&D Program of China (Grant No. 2017YFC1103800).

\section{Disclosure}

The authors report no financial or other conflicts of interest in this work.

\section{References}

1. Liu M, Anderson R, Lan X, et al. Recent advances in the development of nanoparticles for multimodality imaging and therapy of cancer. $\mathrm{Med}$ Res Rev. 2019;40(3):909-930. doi:10.1002/med.21642

2. Holohan C, Van Schaeybroeck S, Longley DB, Johnston PG. Cancer drug resistance: an evolving paradigm. Nat Rev Cancer. 2013;13 (10):714-726. doi:10.1038/nrc3599

3. Wu Y, Li F, Zhang X, et al. Tumor microenvironment-responsive PEGylated heparin-pyropheophorbide -a nanoconjugates for photodynamic therapy. Carbohydr Polym. 2021;255:117490. doi:10.1016/j. carbpol.2020.117490

4. Zhu C, Liu L, Yang Q, Lv F, Wang S. Water-soluble conjugated polymers for imaging, diagnosis, and therapy. Chem Rev. 2012;112 (8):4687-4735. doi:10.1021/cr200263w
5. Hou X, Shou C, He M, et al. A combination of light on gene expression system and tumor microenvironment-responsive nanoparticle delivery system for targeted breast cancer therapy. Acta Pharm Sinica B. 2020;10(9):1741-1753. doi:10.1016/j.apsb.2020.04.010

6. Tao R, Gao M, Liu F, et al. Alleviating the liver toxicity of chemotherapy via $\mathrm{pH}$-responsive hepatoprotective prodrug micelles. ACS Appl Mater Interfaces. 2018;10(26):21836-21846. doi:10. 1021/acsami.8b04192

7. Jia R, Teng L, Gao L, et al. Advances in multiple stimuli-responsive drug-delivery systems for cancer therapy. Int $J$ Nanomed. 2021;16:1525-1551. doi:10.2147/IJN.S293427

8. Blanco E, Shen H, Ferrari M. Principles of nanoparticle design for overcoming biological barriers to drug delivery. Nat Biotechnol. 2015;33(9):941-951. doi:10.1038/nbt.3330

9. Zhao K, Li D, Xu W, et al. Targeted hydroxyethyl starch prodrug for inhibiting the growth and metastasis of prostate cancer. Biomaterials. 2017;116:82-94. doi:10.1016/j.biomaterials.2016.11.030

10. Ekladious I, Colson YL, Grinstaff MW. Polymer-drug conjugate therapeutics: advances, insights and prospects. Nat Rev Drug Discov. 2019;18:273-294. doi:10.1038/s41573-018-0005-0

11. Li J, Anraku Y, Kataoka K. Self-boosting catalytic nanoreactors integrated with triggerable crosslinking membrane networks for initiation of immunogenic cell death by pyroptosis. Angew Chem Int Ed. 2020;59(32):13526-13530. doi:10.1002/anie.202004180

12. Li J, Kataoka K. Chemo-physical strategies to advance the in vivo functionality of targeted nanomedicine: the next generation. $\mathrm{J} \mathrm{Am}$ Chem Soc. 2021;143(2):538-559. doi:10.1021/jacs.0c09029

13. Fang J, Nakamura $H$, Maeda $H$. The EPR effect: unique features of tumor blood vessels for drug delivery, factors involved, and limitations and augmentation of the effect. Adv Drug Deliv Rev. 2011;63 (3):136-351. doi:10.1016/j.addr.2010.04.009

14. Paleos CM, Sideratou Z, Tsiourvas D. Drug delivery systems based on hydroxyethyl starch. Bioconjug Chem. 2017;28(6):1611-1624. doi:10.1021/acs.bioconjchem.7b00400

15. Glover PA, Rudloff E, Kirby R. Hydroxyethyl starch: a review of pharmacokinetics, pharmacodynamics, current products, and potential clinical risks, benefits, and use. J Vet Emerg Crit Care. 2014;24 (6):642-661. doi:10.1111/vec. 12208

16. Xiao $\mathrm{C}, \mathrm{Hu} \mathrm{H}$, Yang $\mathrm{H}$, et al. Colloidal hydroxyethyl starch for tumor-targeted platinum delivery. Nanoscale Adv. 2019; (3):1002-1012. doi:10.1039/c8na00271a

17. Luo Q, Wang P, Miao Y, He H, Tang X. A novel 5-fluorouracil prodrug using hydroxyethyl starch as a macromolecular carrier for sustained release. Carbohydr Polym. 2012;87(4):2642-2647. doi:10.1016/j.carbpol.2011.11.039

18. Li G, Li Y, Tang Y. Hydroxyethyl starch conjugates for improving the stability, pharmacokinetic behavior and antitumor activity of 10-hydroxy camptothecin. Int $J$ Pharm. 2014;471(1-2):234-244. doi:10.1016/j.ijpharm.2014.05.038

19. $\mathrm{Hu} \mathrm{H}$, Li YH, Zhou Q, et al. Redox-sensitive hydroxyethyl starch-doxorubicin conjugate for tumor targeted drug delivery. ACS Appl Mater Interfaces. 2016;8(45):30833-30844. doi:10.1021/ acsami.6b11932

20. Li D, Ding J, Zhuang $X$, et al. Drug binding rate regulates the properties of polysaccharide prodrugs. J Mater Chem B. 2016;4 (30):5167-5177. doi:10.1039/c6tb00991c

21. Li D, Feng X, Chen L, Ding J, Chen X. One-step synthesis of targeted acid-labile polysaccharide prodrug for efficiently intracellular drug delivery. ACS Biomater Sci Eng. 2018;4(2):539-546. doi:10.1021/acsbiomaterials.7b00856

22. Jain RK, Stylianopoulos T. Delivering nanomedicine to solid tumors. Nat Rev Clin Oncol. 2010;7(11):653-664. doi:10.1038/nrclinonc. 2010.139

23. Kim JJ, Tannock IF. Repopulation of cancer cells during therapy: an important cause of treatment failure. Nat Rev Cancer. 2005;5 (7):516-525. doi:10.1038/nrc1650 
24. Minchinton AI, Tannock IF. Drug penetration in solid tumors. Nat Rev Cancer. 2006;6(8):583-592. doi:10.1038/nrc1893

25. Tredan O, Galmarini CM, Patel K, Tannock IF. Drug resistance and the solid tumor microenvironment. $J$ Natl Cancer Inst. 2007;99 (19):1441-1454. doi:10.1093/jnci/djm135

26. Zhang X, Wu Y, Li Z, et al. Glycodendron/pyropheophorbide-a (Ppa)-functionalized hyaluronic acid as a nanosystem for tumor photodynamic therapy. Carbohydr Polym. 2020;247:116749. doi:10.1016/j.carbpol.2020.116749

27. Cai H, Xiang Y, Zeng Y, et al. Cathepsin B-responsive and gadolinium-labeled branched glycopolymer-PTX conjugate-derived nanotheranostics for cancer treatment. Acta Pharm Sinica B. 2021;11(2):544-559. doi:10.1016/j.apsb.2020.07.023

28. Kopecek J. Polymeredrug conjugates: origins, progress to date and future directions. Adv Drug Deliv Rev. 2013;65(1):49-59. doi:10.1016/j.addr.2012.10.014

29. Li J, Ke W, Wang L, et al. Self-sufficing H2O2-responsive nanocarriers through tumor-specific $\mathrm{H} 2 \mathrm{O} 2$ production for synergistic oxidation-chemotherapy. $J$ Control Release. 2016;225:64-74. doi:10.1016/j.jconrel.2016.01.029

30. Lin C, Tong F, Liu R, et al. GSH-responsive SN38 dimer-loaded shapetransformable nanoparticles with iRGD for enhancing chemo-photodynamic therapy. Acta Pharm Sinica B. 2020;10 (12):2348-2361. doi:10.1016/j.apsb.2020.10.009

31. Zhuang J, Chen S, Hu Y, et al. Tumour-targeted and redox-responsive mesoporous silica nanoparticles for controlled release of doxorubicin and an siRNA against metastatic breast cancer. Int $J$ Nanomed. 2021;16:1961-1976. doi:10.2147/IJN.S278724

32. Cheng R, Feng F, Meng F, et al. Glutathione-responsive nano-vehicles as a promising platform for targeted intracellular drug and gene delivery. J Control Release. 2011;152(1):2-12. doi:10.1016/j.jconrel.2011.01.030

33. Kuppusamy P, Li H, Ilangovan G, et al. Noninvasive imaging of tumor redox status and its modification by tissue glutathione levels. Cancer Res. 2002;62(1):307-312.

34. Pang X, Jiang Y, Xiao Q, et al. pH-responsive polymer-drug conjugates: design and progress. J Control Release. 2016;222:116-129. doi:10.1016/j.jconrel.2015.12.024

35. Du J, Du X, Mao C, Wang J. Tailor-made dual pH-sensitive polymer-doxorubicin nanoparticles for efficient anticancer drug delivery. J Am Chem Soc. 2011;133(44):17560-17563. doi:10.1021/ ja207150n

36. Seidi F, Jenjob R, Crespy D. Designing smart polymer conjugates for controlled release of payloads. Chem Rev. 2018;118(7):3965-4036. doi:10.1021/acs.chemrev.8b00006

37. He X, Li J, An S, Jiang C. pH-sensitive drug-delivery systems for tumor targeting. Ther Deliv. 2013;4(12):1499-1510. doi:10.4155/tde.13.120

38. Kanamala M, Wilson WR, Yang M, et al. Mechanisms and biomaterials in $\mathrm{pH}-$ responsive tumour targeted drug delivery: a review. Biomaterials. 2016;85:152-167. doi:10.1016/j.biomaterials.2016.01.061

39. Li J, Dirisala A, Ge Z, et al. Therapeutic vesicular nanoreactors with tumor-specific activation and self-destruction for synergistic tumor ablation. Angew Chem Int Ed. 2017;56(45):14025-14030. doi:10. 1002/anie.201706964

International Journal of Nanomedicine

\section{Publish your work in this journal}

The International Journal of Nanomedicine is an international, peerreviewed journal focusing on the application of nanotechnology in diagnostics, therapeutics, and drug delivery systems throughout the biomedical field. This journal is indexed on PubMed Central, MedLine, CAS, SciSearch ${ }^{\circledR}$, Current Contents ${ }^{\mathbb{B}} /$ Clinical Medicine,
40. Meng F, Zhong Y, Cheng R, et al. pH-sensitive polymeric nanoparticles for tumor-targeting doxorubicin delivery: concept and recent advances. Nanomedicine. 2014;9(3):487-499. doi:10.2217/NNM.13. 212

41. Wang Z, Deng X, Ding J, et al. Mechanisms of drug release in $\mathrm{pH}$-sensitive micelles for tumour targeted drug delivery system: a review. Int $J$ Pharm. 2018;535(1-2):253-260. doi:10.1016/j. ijpharm.2017.11.003

42. Cao X, Luo J, Gong T, et al. Coencapsulated doxorubicin and bromotetrandrine lipid nanoemulsions in reversing multidrug resistance in breast cancer in vitro and in vivo. Mol Pharm. 2015;12 (1):274-286. doi:10.1021/mp500637b

43. Unezaki S, Maruyama K, Hosoda JI, et al. Direct measurement of the extravasation of polyethyleneglycol-coated liposomes into solid tumor tissue by in vivo fluorescence microscopy. Int $J$ Pharm. 1996;144(1):11-17. doi:10.1016/S0378-5173(96)04674-1

44. Chen G, Yan M, Lu QH, Gong M. Effects of two different hydroxyethyl starch solutions (HES200/0.5 Vs. HES130/ 0.4) on the expression of platelet membrane glycoprotein. Acta Anaesthesiol Scand. 2006;50 (9):1089-1094. doi:10.1111/j.1399-6576.2006.01138.x

45. Li Y, Hu H, Zhou Q, et al. $\alpha$-Amylase- and redox-responsive nanoparticles for tumor-targeted drug delivery. ACS Appl Mater Interfaces. 2017;9(22):19215-19230. doi:10.1021/acsami.7b04066

46. Yu C, Zhou Q, Xiao F, et al. Enhancing doxorubicin delivery toward tumor by hydroxyethyl starch-g-polylactide partner nanocarriers. ACS Appl Mater Interfaces. 2017;9(12):10481-10493. doi:10.1021/ acsami.7b00048

47. Wang A, Langer R, Farokhzad OC. Nanoparticle delivery of cancer drugs. Annu Rev Med. 2012;63(1):185-198. doi:10.1146/annurevmed-040210-162544

48. Bertrand N, Wu J, Xu X, et al. Cancer nanotechnology: the impact of passive and active targeting in the era of modern cancer biology. $A d v$ Drug Deliv Rev. 2014;66:2-25. doi:10.1016/j.addr.2013.11.009

49. Chuan X, Qin S, Lin J, et al. Novel free-paclitaxel-loaded redox-responsive nanoparticles based on a disulfide-linked poly(ethylene glycol)-drug conjugate for intracellular drug delivery: synthesis, characterization, and antitumor activity in vitro and in vivo. $\mathrm{Mol}$ Pharm. 2014;11(10):3656-3670. doi:10.1021/mp500399j

50. Wu H, Hu H, Wan J, et al. Hydroxyethyl starch stabilized polydopamine nanoparticles for cancer chemotherapy. Chem Eng J. 2018;349:129-145. doi:10.1016/j.cej.2018.05.082

51. Carvalho C, Santos RX, Cardoso S, et al. Doxorubicin: the good, the bad and the ugly effect. Curr Med Chem. 2009;16(25):3267-3285. doi:10.2174/092986709788803312

52. Deng Y, Huang L, Yang H, et al. Cyanine-anchored silica nanochannels for light-driven synergistic thermo-chemotherapy. Small. 2017;13(6):1602747. doi:10.1002/smll.201602747

53. Adwas AA, Elkhoely AA, Kabel AM, et al. Anti-cancer and cardioprotective effects of indol-3-carbinol in doxorubicin-treated mice. $J$ Infect Chemother. 2016;22(1):36-43. doi:10.1016/j.jiac.2015.10.001

54. Khan G, Haque SE, Anwer T, et al. Cardioprotective effect of green tea extract on doxorubicin-induced cardiotoxicity in rats. Acta Pol Pharm. 2014;71:861-868.
Journal Citation Reports/Science Edition, EMBase, Scopus and the Elsevier Bibliographic databases. The manuscript management system is completely online and includes a very quick and fair peer-review system, which is all easy to use. Visit http://www.dovepress.com/ testimonials.php to read real quotes from published authors. 